\title{
30. EAST PACIFIC RISE, GALAPAGOS SPREADING CENTER AND SIQUEIROS FRACTURE ZONE, DEEP SEA DRILLING PROJECT LEG 54: HYGROMAGMAPHILE ELEMENTS- A COMPARISON WITH THE NORTH ATLANTIC
}

\author{
J. L. Joron ${ }^{2}$, L. Briqueu ${ }^{3}$, H. Bougault ${ }^{4}$, and M. Treuil ${ }^{2}$
}

\begin{abstract}
Basalt samples collected on DSDP Leg 54 are fresh, with some showing extensive $\mathrm{Fe}$ - and Ti-enrichment. Samples from many sites were contaminated with $\mathrm{Co}, \mathrm{Ta}$, and, to a lesser extent, $\mathrm{Nb}$, because they were powdered using a tungsten carbide shatter box onboard ship. $\mathrm{Y} / \mathrm{Tb}, \mathrm{Zr} / \mathrm{Hf}$, and (for uncontaminated samples) $\mathrm{Nb} / \mathrm{Ta}$ ratios are similar to those of North Atlantic basalts and chondritic meteorites; these ratios are probably characteristic of the primordial mantle. The $\mathrm{La} / \mathrm{Ta}$ and $\mathrm{Y} / \mathrm{La}$ ratios indicate that the Leg 54 eastern Pacific basalts were derived from "depleted" mantle similar to the source of North Atlantic basalts drilled on DSDP Leg $45\left(22^{\circ} \mathrm{N}\right.$; Miocene crust) and Legs 51 through $53\left(25^{\circ} \mathrm{N}\right.$; Cretaceous crust).
\end{abstract}

\section{INTRODUCTION}

DSDP Leg 54 was the first IPOD leg in the North Pacific dealing with the oceanic crust. Several sites were drilled: the western flank of the East Pacific Rise (EPR) at $9^{\circ} \mathrm{N}$ (Figure 1), Sites 420,421 ( $\sim 3.5$ m.y. old), 423 ( $\sim 1.2$ m.y. old), 422,428 ( $\sim 2$ m.y. old $)$, and $429(\sim 4.5$ m.y. old); the Galapagos spreading center at $0^{\circ} \mathrm{N}$, $86^{\circ} \mathrm{W}$, four holes at Site 424, and Site $425(62 \mathrm{~km}$ north of the Galapagos spreading center axis); and the Siqueiros fracture zone, Site $427\left(8^{\circ} \mathrm{N}, \sim 5 \mathrm{~m} . \mathrm{y}\right.$. old $)$.

Samples from this drilling program should give us a picture of the variability of basalts produced in this area and a basis of comparison with results obtained in the North Atlantic. Our investigations focused on $\mathrm{Y} / \mathrm{Tb}$, $\mathrm{Zr} / \mathrm{Hf}$, and $\mathrm{Nb} / \mathrm{Ta}$ ratios, shown to be constant for DSDP and dredged samples in the North Atlantic, and on the $\mathrm{La} / \mathrm{Ta}$ ratio.

In the North Atlantic, on the basis of $\mathrm{La} / \mathrm{Ta}$ ratios, we can identify two main mantle sources for the basalts of the oceanic crust. The first source is characterized by a $\mathrm{La} / \mathrm{Ta}$ ratio equal to 18 , which represents the so-called "typical MORB tholeiites."' Basalts drilled at Sites 395 and 396 on either side of the Mid-Atlantic Ridge during Legs 45 and 46 have this ratio. The second source is characterized by a La/Ta ratio equal to 9 and represents the basalts of the oceanic islands and seamounts and also of parts of the ridges near these islands. Basalts drilled and dredged in the FAMOUS area at $36^{\circ} \mathrm{N}$, at

\footnotetext{
${ }^{1}$ Contribution no. 530 du Departement Scientifique, Centre Océanologique de Bretagne.

2 Institut de Physique du Globe, Laboratoire de Geochimie, 4. place Jussieu, 75230 Paris Cedex 05, France.

${ }^{3}$ Université des Sciences et Techniques du Languedoc, Place Eugène Bataillon, 34000 Montpellier, France.

${ }_{4}$ Centre Océanologique de Bretagne, B.P. 337, 29273 Brest Cedex, France.
}

$45^{\circ} \mathrm{N}$, and near Iceland, during Leg 49 , have this ratio (see Figure 2).

We also deal with a contamination problem caused by powdering the samples in a tungsten carbide shatter box onboard the Glomar Challenger.

\section{ANALYTICAL CHEMISTRY: THE CONTAMINATION PROBLEM}

Analytical procedures (X-ray fluorescence [XRF] both onboard ship and on shore and neutron activation analysis) have been described previously (Bougault, 1977; and Bougault et al., 1978). As on several previous legs, we have systematically analyzed trace elements in samples selected onboard ship for shipboard analysis. Unfortunately, on Leg 54 the oil tank of the generator of the XRF unit burned out. This represented the first major breakdown of our shipboard equipment since we started analysis onboard the Glomar Challenger (Leg 37) and, consequently, most of our major-oxide results were obtained on shore. In addition, the agate mortar was cracked, pitted, and was therefore unusable. Consequently, we prepared the powders onboard ship using the shipboard tungsten carbide shatter box. We knew in advance that this procedure would lead to cobalt contamination but did not know the extent of the problem. Although some results may not be suitable for geochemical interpretation, we measured the same elements as on previous cruises, to determine what other elements (if any) were involved in the contamination.

Hole 427 samples (Table 13b) provided a good opportunity to ascertain which elements were contaminated by use of the tungsten carbide shatter box, as some specimens were ground in this manner, others by means of the agate mortar. The former evinced a high tungsten concentration. We confirmed the anticipated contamination of cobalt: values as high as $80 \mathrm{ppm}$ in contaminated samples occur, whereas the average in uncontaminated samples is about $46 \mathrm{ppm}$, a typical value in oceanic 


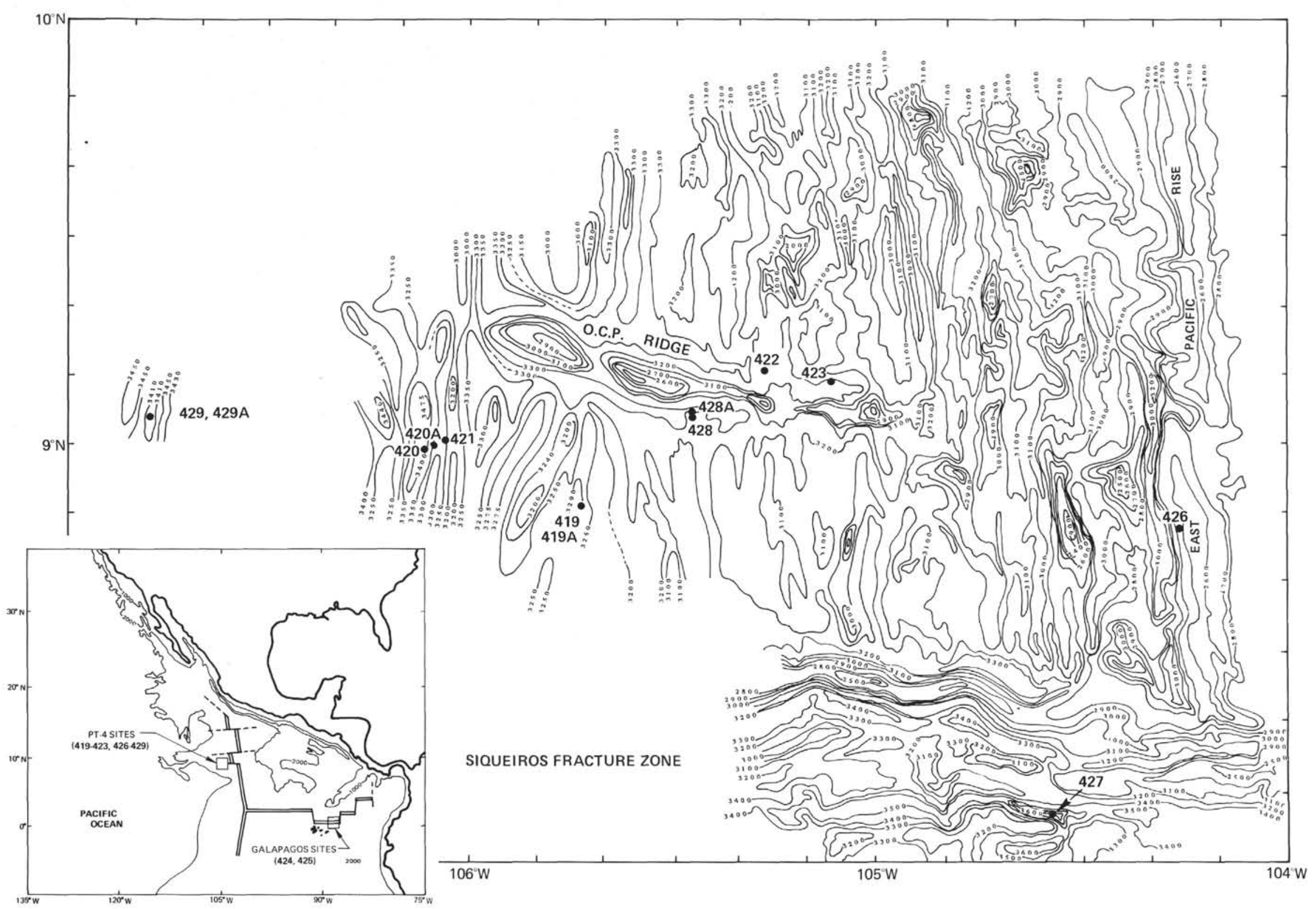

Figure 1. Sites drilled on Deep Sea Drilling Project Leg 54. Contours in meters. 


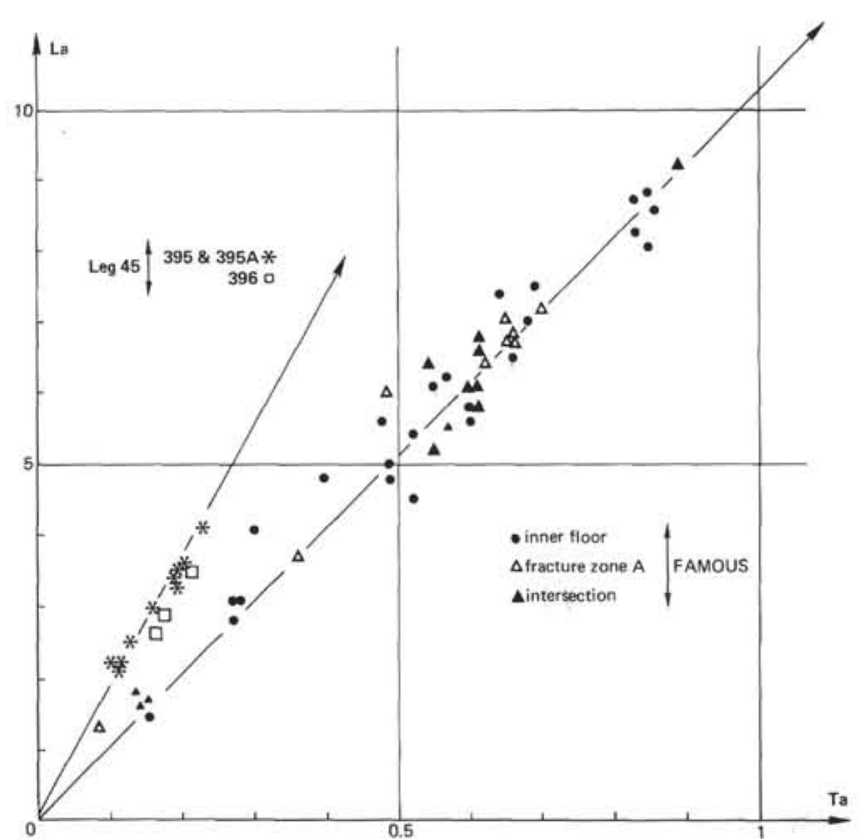

Figure 2. La (ppm) versus Ta (ppm) for basalts drilled and dredged on Leg 49. Symbols for this and Figures 3 through 8 are as follows: - Hole $420 ;+$ : Hole $421 ; \times$ : Hole $422 ; \triangle:$ Hole $423 ; \square$ : Hole 424 and $424 \mathrm{~A}$; : Holes $424 \mathrm{~B}$ and $424 \mathrm{C}$; $\star$ : Hole 425 ; 战 : Hole $427 ; \boldsymbol{\Lambda}:$ Holes 428 and $428 A$; and $\bigcirc:$ Hole 429 A.

tholeiites. Correspondingly, very high tantalum concentrations ( 3 to $5 \mathrm{ppm}$ ) occur in contaminated samples, whereas the average contents in uncontaminated samples is $0.37 \mathrm{ppm}$. Niobium is also subject to contamination but to a lesser extent. Values of about $9.3 \mathrm{ppm}$ occur in contaminated samples, whereas the average value in uncontaminated samples is $7.2 \mathrm{ppm}$. The other elements investigated - Hf, Th, Sc, V, Cr, Ni, Rb, Sr, Y, and $\mathrm{Zr}$ - showed no evidence of contamination.

These observations are confirmed on the one hand by samples of Holes 420 through 425 (Tables $1 \mathrm{~b}, 2 \mathrm{~b}, 3 \mathrm{~b}$, $4 \mathrm{~b}$, and $12 \mathrm{~b}$ ), all of them ground with the tungsten carbide shatter box, and on the other hand by the samples of Holes 428 and 429 (Tables 5a, 6b, and 7b) which were ground with the agate mortar. Thus, it appears that the tungsten carbide shatter box used on Leg 54 causes considerable contamination of cobalt and tantalum, and, to a lesser extent, niobium. This evaluation is, of course, made by comparison with the concentrations of uncontaminated samples presently studied, which are Co $\sim 45$ $\mathrm{ppm}, \mathrm{Ta} \sim 0.3 \mathrm{ppm}$, and $\mathrm{Nb} \sim 7 \mathrm{ppm}$. Contamination of $\mathrm{Ta}$ and $\mathrm{Nb}$ results from impurities in the tungsten, that of cobalt because this metal is used as a binder for the tungsten carbide.

\section{RESULTS}

The tables are arranged not by hole or site numbers but according to drilling area. Tables 1 through 7 are concerned with the western flank of the EPR, Holes $420,421,423,422,428,428 \mathrm{~A}$, and 429 , respectively. Table 8 through 12 relate to the Galapagos spreading center - Holes 424, 424A, 424B, 424C, and 425, respectively. Table 13 relates to the Siqueiros transform fault (Hole 427).

In the first place, all of the samples have low loss on ignition, and are considered reasonably fresh. Only one significant instance was found, in Hole 428 (Tables 6a and $6 \mathrm{~b}$ ), of alkali metals being sensitive to alteration effects (Bougault et al., 1978; Joron et al., in press). From the oxide analyses in 17 samples it appears that the basalts of this hole are homogeneous. Alkali-metal concentrations are low $\left(\mathrm{K}_{2} \mathrm{O}<0.2 \%, \mathrm{Rb}<3 \mathrm{ppm}\right.$, and Cs $<0.05 \mathrm{ppm}$ ), except for Sections 428A-1-4, No. 9 and 428A-2-1, No. $4\left(\mathrm{~K}_{2} \mathrm{O}: 0.44\right.$ and $0.29 \%$, Rb: 6.7 and 3.9 ppm, and Cs: 0.28 and $0.17 \mathrm{ppm}$, respectively). This enrichment results from a moderate alteration effect, comparable to alteration observed in Hole 395 of Leg 45 (Bougault et al., 1978). This variation is by no means to be interpreted as the result of magmatic processes.

With respect to both major oxide and trace elements, despite the number of holes drilled on the western flank of the EPR, the range of basaltic types is rather limited. Choosing $\mathrm{TiO}_{2}$ as an indicator, three types of hygromagmaphile element increases can be distinguished: Type 1 (Holes 428, 428A, and 422) with $\mathrm{TiO}_{2}$ about 1.45 per cent; Type 2 (Section 10-2, No. 3 of Hole 422, Holes 420,423 , and 421) with $\mathrm{TiO}_{2}$ about 2 per cent and Type 3 (first two samples of Hole 421) with $\mathrm{TiO}_{2}$ about 2.5 per cent.

Within Type 1, four samples (Sections 9-1, 9-3, 9-4, and 9-5 in Hole 422; Table 2a) have higher $\mathrm{Al}_{2} \mathrm{O}_{3}$ $(15.8 \%$ instead of $14.2 \%)$ and Sr (174 ppm instead of $137 \mathrm{ppm}$ ) than other samples within this group. Enrichment in plagioclase and clinopyroxene phenocrysts could account for the variations observed, but $\mathrm{Y}, \mathrm{Zr}, \mathrm{Tb}$, and $\mathrm{Hf}$ are the same as in other samples of the group. Sr and Th are enriched, and $\mathrm{Sc}$ is depleted (and remains somewhat problematic).

Within Type 2, some significant differences can be observed; that is, Section 10-2, No. 3 of Hole 422 and Hole 420 have $\mathrm{TiO}_{2}$ contents of about 1.92 per cent, Hole $423 \mathrm{TiO}_{2}$ contents of about 2.05 per cent, and Hole $421 \mathrm{TiO}_{2}$ contents of about 2.13 per cent. These differences are confirmed by other elements (Figures 3 , 4 , and 5).

Basalts having at least two different compositions were obtained in Hole 429 (4.5 m.y. old); they appear to be similar to Type 1 basalts.

From the Galapagos rift, samples recovered in Holes $424,424 \mathrm{~A}, 424 \mathrm{~B}$, and $424 \mathrm{C}$ appear to be identical. They are characterized by low $\mathrm{Al}_{2} \mathrm{O}_{3}$ values $(12.6 \%)$, high $\mathrm{Fe}_{2} \mathrm{O}_{3}$ values $(15.1 \%)$, and very low strontium concentrations $(66 \mathrm{ppm})$ as compared with typical values of oceanic tholeiites. $\mathrm{TiO}_{2}$ is about 1.87 per cent. Two main groups are present in Hole 425 drilled at $62 \mathrm{~km}$ north of the spreading center: they have $\mathrm{TiO}_{2}$ values of 0.98 per cent and 1.19 per cent (one other sample has $\mathrm{TiO}_{2}=1.42 \%$ ). A feature in common with the Site 424 samples is the low value of strontium $(50 \mathrm{ppm})$. From the data available, this low strontium concentration seems to be a characteristic feature of the Galapagos rift. 
TABLE 1a

Hole 420 (EPR, $9^{\circ} \mathrm{N}, \sim 3.5$ m.y. old): Major Oxides

\begin{tabular}{|c|c|c|c|c|c|c|c|c|c|c|c|c|}
\hline $\begin{array}{c}\text { Sample } \\
\text { (Interval in } \mathrm{cm} \text { ) }\end{array}$ & $\mathrm{SiO}_{2}$ & $\mathrm{Al}_{2} \mathrm{O}_{3}$ & $\mathrm{Fe}_{2} \mathrm{O}_{3}$ & $\mathrm{MnO}$ & $\mathrm{MgO}$ & $\mathrm{CaO}$ & $\mathrm{Na}_{2} \mathrm{O}$ & $\mathrm{K}_{2} \mathrm{O}$ & $\mathrm{TiO}_{2}$ & $\mathrm{P}_{2} \mathrm{O}_{5}$ & LOI & Total \\
\hline $14-1,0-8$ & 50.43 & 13.77 & 12.40 & 0.20 & 7.18 & 11.21 & 2.55 & 0.16 & 1.94 & 0.19 & 0.14 & 100.17 \\
\hline $15-1$ (No. 4) & 50.38 & 13.99 & 11.63 & 0.19 & 6.69 & 11.31 & 2.75 & 0.16 & 1.92 & 0.16 & 1.01 & 100.19 \\
\hline $16-1,2-10$ & 50.65 & 13.87 & 12.47 & 0.20 & 7.02 & 11.14 & 2.62 & 0.30 & 1.92 & 0.17 & 0.38 & 100.74 \\
\hline $17-1,27-30$ & 50.67 & 14.28 & 11.00 & 0.19 & 6.30 & 11.20 & 2.80 & 0.43 & 1.99 & 0.16 & 1.10 & 100.09 \\
\hline
\end{tabular}

TABLE 1b

Hole 420 (EPR, $9^{\circ} \mathrm{N}, \sim 3.5$ m.y. old): Trace Elements

\begin{tabular}{|c|c|c|c|c|c|c|c|c|c|c|c|c|c|c|c|c|c|c|c|c|c|c|c|}
\hline $\begin{array}{c}\text { Sample } \\
\text { (Interval in } \mathrm{cm} \text { ) }\end{array}$ & $\begin{array}{l}\mathrm{Sc} \\
\mathrm{NA}\end{array}$ & $\begin{array}{c}\mathrm{Ti} \\
\text { XRF }\end{array}$ & $\begin{array}{c}\mathrm{V} \\
\text { XRF }\end{array}$ & $\begin{array}{c}\mathrm{Cr} \\
\mathrm{XRF}\end{array}$ & XRF & NAA & $\begin{array}{c}\mathrm{Ni} \\
\mathrm{NAA}\end{array}$ & $\begin{array}{c}\mathrm{Rb} \\
\mathrm{XRF}\end{array}$ & $\begin{array}{c}\text { St } \\
\text { XRF }\end{array}$ & $\begin{array}{c}\mathrm{Y} \\
\text { XRF }\end{array}$ & $\begin{array}{c}\mathrm{Zr} \\
\mathrm{XRF}\end{array}$ & $\begin{array}{r}\mathrm{Nb} \\
\mathrm{XRF}\end{array}$ & $\begin{array}{c}\mathrm{Sb} \\
\text { NAA }\end{array}$ & $\begin{array}{c}\mathrm{Cs} \\
\text { NAA }\end{array}$ & $\begin{array}{c}\mathrm{Ba} \\
\text { NAA }\end{array}$ & $\begin{array}{c}\mathrm{La} \\
\mathrm{NAA}\end{array}$ & $\begin{array}{c}\text { Eu } \\
\text { NAA }\end{array}$ & $\begin{array}{c}\mathrm{Tb} \\
\text { NAA }\end{array}$ & $\begin{array}{c}\text { Hf } \\
\text { NAA }\end{array}$ & $\begin{array}{c}\mathrm{Ta} \\
\text { NAA }\end{array}$ & $\begin{array}{c}\text { Th } \\
\text { NAA }\end{array}$ & $\begin{array}{c}\text { U } \\
\text { NAA }\end{array}$ & $\begin{array}{c}\text { W } \\
\text { NAA }\end{array}$ \\
\hline $14-1,0-8$ & 43 & 11640 & 363 & 160 & 92 & 93 & 64 & 0.8 & 111 & 50 & 134 & 8.1 & 0.13 & - & - & 4.4 & 1.66 & 1.66 & 3.6 & 5.6 & 0.26 & - & 857 \\
\hline Section 15-1, (No. 4) & 43 & 11520 & 364 & 166 & 174 & 177 & 91 & 4 & 126 & 51 & 133 & 7.3 & - & 0.12 & 9 & 4. & 1.67 & 1.67 & 3.5 & 6.9 & 0.26 & - & 1040 \\
\hline $16-1,2-10$ & 43 & 11520 & 364 & 162 & 107 & 104 & 61 & 4 & 118 & 50 & 122 & 8.4 & 0.06 & 0.11 & 5 & 3. & & 1.59 & 3.4 & 6.1 & 0.20 & - & 933 \\
\hline $17-1,27-30$ & 45 & 11940 & 391 & 176 & 115 & 117 & 102 & 7 & 131 & 51 & 130 & 6.3 & - & - & - & 4.1 & 1.73 & 1.73 & 3.6 & 6.58 & 0.22 & - & 864 \\
\hline
\end{tabular}

TABLE 2a

Hole 421 (EPR, $9^{\circ} \mathrm{N}, \sim 3.5$ m.y. old): Major Oxides

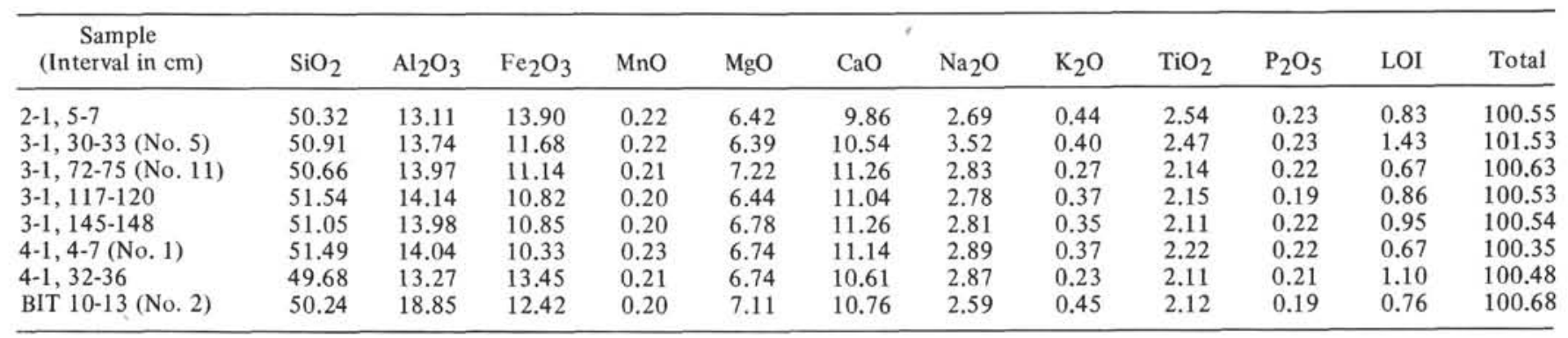

TABLE 2b

Hole 421 (EPR, $9^{\circ} \mathrm{N}, \sim 3.5$ m.y. old): Trace Elements

\begin{tabular}{|c|c|c|c|c|c|c|c|c|c|c|c|c|c|c|c|c|c|c|c|c|c|c|c|}
\hline $\begin{array}{c}\text { Sample } \\
\text { (Interval in } \mathrm{cm} \text { ) }\end{array}$ & $\begin{array}{l}\mathrm{Sc} \\
\text { NA }\end{array}$ & $\underset{\text { XRF }}{\mathrm{Ti}}$ & $\begin{array}{l}\mathrm{V} \\
\mathrm{XRF}\end{array}$ & $\begin{array}{c}\mathrm{Cr} \\
\mathrm{XRF}\end{array}$ & XRF & ${ }^{\circ}$ & $\begin{array}{c}\mathrm{Ni} \\
\text { NAA }\end{array}$ & $\begin{array}{c}\mathrm{Rb} \\
\mathrm{XRF}\end{array}$ & $\begin{array}{c}\text { Sr } \\
\text { XRF }\end{array}$ & $\begin{array}{c}\mathrm{Y} \\
\mathrm{XRF}\end{array}$ & $\begin{array}{c}\mathrm{Zr} \\
\mathrm{XRF}\end{array}$ & $\begin{array}{c}\mathrm{Nb} \\
\mathrm{XRF}\end{array}$ & $\begin{array}{c}\text { Sb } \\
\text { NAA }\end{array}$ & $\begin{array}{c}\text { Cs } \\
\text { NAA }\end{array}$ & $\begin{array}{c}\mathrm{Ba} \\
\text { NAA }\end{array}$ & $\begin{array}{c}\mathrm{La} \\
\text { NAA }\end{array}$ & $\begin{array}{c}\text { Eu } \\
\text { NAA }\end{array}$ & $\begin{array}{l}\text { Tb } \\
\text { NAA }\end{array}$ & $\begin{array}{l}\mathrm{Hf} \\
\text { NAA }\end{array}$ & $\begin{array}{c}\mathrm{Ta} \\
\text { NAA }\end{array}$ & $\begin{array}{l}\text { Th } \\
\text { NAA }\end{array}$ & $\begin{array}{c}\text { U } \\
\text { NAA }\end{array}$ & $\begin{array}{c}\text { W } \\
\text { NAA }\end{array}$ \\
\hline $2-1,5-7$ & 44 & 15240 & 453 & 78 & 100 & 96 & 48 & 5 & 118 & 68 & 177 & 8.9 & 0.08 & 0.10 & - & - & 2.09 & 1.18 & 4.8 & 5.12 & 0.28 & 0.28 & - \\
\hline 3-1, 30-33 (No. 5) & 45 & 14820 & 447 & 90 & 102 & 103 & 55 & 3 & 146 & 67 & 167 & 8.3 & - & 0.04 & 27 & - & 2.07 & 1,31 & 4.65 & 2.89 & 0.29 & 0.29 & - \\
\hline $3-1,72-75($ No, 11$)$ & 44 & 12840 & 375 & 165 & 202 & 207 & 61 & 3 & 135 & 57 & 147 & 6.5 & 0.04 & 0.10 & 32 & - & 1.89 & 1.09 & 3.85 & 4.4 & 0.35 & 0.35 & - \\
\hline $3-1,117-120$ (No. 17) & 45 & 12900 & 415 & 164 & 102 & 106 & 93 & 2 & 133 & 58 & 151 & 7.1 & 0.03 & 0.07 & 27 & - & 1.99 & 1.14 & 4.03 & 4.34 & 0.22 & 0.22 & - \\
\hline $3-1,145-148$ & 45 & 12660 & 380 & 135 & 125 & 130 & 45 & 3 & 135 & 56 & 157 & 6.9 & 0.04 & 0.15 & 25 & - & 1.91 & 1.07 & 3.86 & 3.72 & 0.21 & 0.21 & - \\
\hline $4-1,4-7$ (No. 1) & 45 & 13320 & 420 & 147 & 126 & 133 & 82 & 1 & 138 & 62 & 159 & 8.8 & - & - & 44 & - & 2.06 & 1.21 & 4.1 & 6.03 & 0.29 & 0.29 & - \\
\hline $4-1,32 \cdot 36$ & 42 & 12660 & 391 & 131 & 167 & 165 & 56 & 3 & 110 & 59 & 149 & 11.6 & 0.05 & 0.03 & - & - & 1.97 & .099 & 3.70 & 11.61 & 0.22 & 0.22 & - \\
\hline BIT $10-13$ (No. 2) & 43 & 12720 & 388 & 130 & 92 & 90 & 53 & - & 127 & 56 & 156 & 7.8 & 0.05 & 0.20 & 13 & - & 1.62 & 1.01 & 3.95 & 3.46 & 0.24 & 0.24 & \\
\hline
\end{tabular}

Hole 427 which was drilled in the Siqueiros fracture zone consists of one single homogeneous unit. Although the $\mathrm{Al}_{2} \mathrm{O}_{3}$ content is rather low $(13.3 \%), \mathrm{Sr}$ concentrations $(\sim 125 \mathrm{ppm})$ correspond to a typical $\mathrm{Sr}$ value in oceanic tholeiites. These samples are similar to Type 3 basalts, as defined by the two uppermost samples of Hole 421 on the western flank of the EPR.

\section{HYGROMAGMAPHILE ELEMENTS}

In all samples recovered by drilling in the Atlantic Ocean (as well as those recovered during the FAMOUS operation), for each pair $\mathrm{Y}-\mathrm{Tb}, \mathrm{Zr}-\mathrm{Hf}$, and $\mathrm{Nb}-\mathrm{Ta}$, one element has been shown not to fractionate with respect to the other (Bougault et al., in press; Joron et al., in press). Thus, the ratios of these elements are constant or nearly constant for each pair, and close to chondritic ratios.
Because of the contamination problem, $\mathrm{Nb}$ and $\mathrm{Ta}$ data are available only for two EPR sites (Holes 428, $428 \mathrm{~A}$, and 429A) and for the Siqueiros fracture zone (Hole 427). The parallel lines on Figure 6 show a major portion of the range of North Atlantic data (absolute values go up to $7.8 \mathrm{ppm}$ for $\mathrm{Ta}$ and $93 \mathrm{ppm}$ for $\mathrm{Nb}$ at $45^{\circ} \mathrm{N}$ ); data of Leg 54 plot on the same line.

All $\mathrm{Zr}$-Hf and Y-Tb data can be used since no contamination has been observed for these elements. As with $\mathrm{Nb}-\mathrm{Ta}$, all the $\mathrm{Zr}-\mathrm{Hf}$ data of Leg 54 plot in the range defined by the samples of the Atlantic Ocean (Figure 3). On the Y-Tb diagram (Figure 4) the data plot on a line the slope of which is a little higher than the average slope of the Atlantic. Since this difference can result from a small systematic difference of 2 to $3 \mathrm{ppm}$ in $\mathrm{Y}$ or 0.1 to $0.2 \mathrm{ppm}$ in $\mathrm{Tb}$, we do not state that the Leg 54 line is distinct from the North Atlantic line. 
TABLE 3a

Hole 422 (EPR, $9^{\circ} \mathrm{N}, \sim 2$ m.y. old): Major Oxides

\begin{tabular}{|c|c|c|c|c|c|c|c|c|c|c|c|c|}
\hline $\begin{array}{c}\text { Sample } \\
\text { (Interval in } \mathrm{cm} \text { ) }\end{array}$ & $\mathrm{SiO}_{2}$ & $\mathrm{Al}_{2} \mathrm{O}_{3}$ & $\mathrm{Fe}_{2} \mathrm{O}_{3}$ & $\mathrm{MnO}$ & $\mathrm{MgO}$ & $\mathrm{CaO}$ & $\mathrm{Na}_{2} \mathrm{O}$ & $\mathrm{K}_{2} \mathrm{O}$ & $\mathrm{TiO}_{2}$ & $\mathrm{P}_{2} \mathrm{O}_{5}$ & LOI & Total \\
\hline 5-1, 23-26 (No. 5) & 50.26 & 14.03 & 10.59 & 0.18 & 7.66 & 12.04 & - & 0.11 & 1.44 & 0.16 & 0.33 & 96.81 \\
\hline $7-1,6-10$ & 50.44 & 14.26 & 10.46 & 0.17 & 7.80 & 11.95 & 2.61 & 0.17 & 1.46 & 0.15 & 0.76 & 100.23 \\
\hline $7-1,20-25$ & 50.49 & 14.17 & 10.61 & 0.17 & 7.83 & 11.98 & 2.39 & 0.13 & 1.44 & 0.17 & 0.76 & 100.14 \\
\hline $7-1,89-92$ (No. 7 ) & 50.55 & 14.30 & 10.70 & 0.18 & 7.82 & 12.02 & 2.75 & 0.11 & 1.44 & 0.16 & 0.19 & 100.21 \\
\hline $7-1,122-131$ & 50.56 & 14.30 & 10.56 & 0.17 & 7.78 & 12.09 & 2.59 & 0.18 & 1.43 & 0.14 & 0.48 & 100.28 \\
\hline Section 7-2, (No. 3) & 50.74 & 14.28 & 10.58 & 0.18 & 8.04 & 12.00 & 2.70 & 0.13 & 1.45 & 0.15 & 0.21 & 100.46 \\
\hline $8-5,23-26$ (No. 5$)$ & - & - & - & - & - & - & 2.77 & - & - & - & - & - \\
\hline $8-5,132-135$ (No. 14) & 50.73 & 14.32 & 10.46 & 0.17 & 8.21 & 11.99 & 2.74 & 0.10 & 1.45 & 0.17 & 0.14 & 100.46 \\
\hline $9-1,70-73$ (No. 4c) & 50.28 & 13.99 & 10.65 & 0.17 & 7.87 & 11.92 & 2.71 & 0.09 & 1.43 & 0.15 & 1.04 & 100.30 \\
\hline $9-2,135-138$ (No. 6) & 49.76 & 15.96 & 9.80 & 0.19 & 8.54 & 11.72 & 2.83 & 0.22 & 1.40 & 0.20 & 0.67 & 101.30 \\
\hline 9-3, 91-94 (No. 6c) & 48.59 & 15.50 & 9.38 & 0.19 & 8.45 & 11.59 & 2.85 & 0.23 & 1.38 & 0.18 & 1.33 & 99.66 \\
\hline $9-4,41-45$ (No. 3b) & 49.64 & 15.96 & 7.67 & 0.15 & 8.73 & 11.67 & 2.80 & 0.25 & 1.40 & 0.20 & 0.67 & 101.14 \\
\hline $9-5,72-75$ (No. 6) & 49.77 & 15.83 & 9.79 & 0.15 & 8.63 & 11.68 & 2.83 & 0.21 & 1.43 & 0.18 & 0.33 & 100.82 \\
\hline $10-2,19-25$ (No. 3 ) & 50.39 & 13.92 & 11.84 & 0.20 & 7.09 & 11.72 & 2.94 & 0.31 & 1.88 & 0.21 & 0 & 100.50 \\
\hline $10-1,12 ?$ & - & - & - & - & - & - & 2.41 & - & - & - & - & - \\
\hline
\end{tabular}

TABLE $3 b$

Hole 422 (EPR, $9^{\circ} \mathrm{N}, \sim 2$ m.y. old): Trace Elements

\begin{tabular}{|c|c|c|c|c|c|c|c|c|c|c|c|c|c|c|c|c|c|c|c|c|c|c|c|}
\hline \multirow{2}{*}{$\begin{array}{c}\text { Sample } \\
\text { (Interval in } \mathrm{cm} \text { ) }\end{array}$} & \multirow{2}{*}{$\begin{array}{l}\mathrm{Sc} \\
\mathrm{NA}\end{array}$} & \multirow{2}{*}{$\begin{array}{c}\mathrm{Ti} \\
\mathrm{XRF}\end{array}$} & \multirow{2}{*}{$\begin{array}{c}\mathrm{V} \\
\mathrm{XRF}\end{array}$} & \multirow{2}{*}{$\underset{\mathrm{XRF}}{\mathrm{Cr}}$} & \multicolumn{2}{|c|}{$\mathrm{Co}$} & \multirow{2}{*}{$\begin{array}{c}\mathrm{Ni} \\
\mathrm{NAA}\end{array}$} & \multirow{2}{*}{$\begin{array}{l}\mathrm{Rb} \\
\mathrm{XRF}\end{array}$} & \multirow{2}{*}{$\begin{array}{c}\text { Sr } \\
\text { XRF }\end{array}$} & \multirow{2}{*}{$\begin{array}{c}\text { Y } \\
\text { XRF }\end{array}$} & \multirow{2}{*}{$\begin{array}{c}\mathrm{Zr} \\
\mathrm{XRF}\end{array}$} & \multirow{2}{*}{$\begin{array}{c}\mathrm{Nb} \\
\mathrm{XRF}\end{array}$} & \multirow{2}{*}{$\begin{array}{l}\mathrm{Sb} \\
\text { NAA }\end{array}$} & \multirow{2}{*}{$\begin{array}{l}\text { Cs } \\
\text { NAA }\end{array}$} & \multirow{2}{*}{$\begin{array}{l}\text { Ba } \\
\text { NAA }\end{array}$} & \multirow{2}{*}{$\stackrel{\mathrm{La}}{\mathrm{NAA}}$} & \multirow{2}{*}{$\begin{array}{c}\text { Eu } \\
\text { NAA }\end{array}$} & \multirow{2}{*}{$\begin{array}{l}\text { Tb } \\
\text { NAA }\end{array}$} & \multirow{2}{*}{$\begin{array}{c}\mathrm{Hf} \\
\text { NAA }\end{array}$} & \multirow{2}{*}{$\begin{array}{l}\text { Ta } \\
\text { NAA }\end{array}$} & \multirow{2}{*}{$\begin{array}{l}\text { Th } \\
\text { NAA }\end{array}$} & \multirow{2}{*}{$\underset{\text { NAA }}{U}$} & \multirow{2}{*}{$\stackrel{W}{\text { NAA }}$} \\
\hline & & & & & XRF & $\mathrm{NAA}$ & & & & & & & & & & & & & & & & & \\
\hline $7-1,6-10$ & - & 8760 & 278 & 231 & - & - & - & 0.5 & 137 & 42 & 97 & 8.3 & - & - & - & - & 1.31 & 0.69 & - & - & - & - & - \\
\hline $7-1,20-25$ & 40 & 8640 & 286 & 236 & 130 & 129 & 69 & 1 & 138 & 40 & 108 & 5.4 & 0.01 & .04 & - & 3.2 & - & - & 2.34 & 4.73 & 0.10 & - & 757 \\
\hline Section $89-92,(\mathrm{No}, \mathrm{zc})$ & - & 8640 & 296 & 237 & 299 & - & - & 1 & 138 & 37 & 156 & 5.5 & - & - & - & - & 1.36 & 0.71 & - & - & - & - & - \\
\hline $7-1,122-131$ & 41 & 8580 & 295 & 281 & - & 90 & 81 & 0.2 & 146 & 36 & - & 5.7 & 0.06 & - & - & 2.9 & 1.41 & 0.62 & 2.41 & 4.37 & 0.09 & 0.34 & 664 \\
\hline Section $7-2,($ No. 3$)$ & 41 & 8700 & 276 & 237 & - & 98 & 75 & 0.1 & 140 & 37 & 97 & 5.5 & 0.02 & - & - & 3.2 & 1.3 & 0.72 & 2.63 & 5.98 & 0.09 & 0.21 & 836 \\
\hline $8-5,23-26$ (No. 5$)$ & 41 & $=$ & - & - & - & 108 & 78 & - & - & - & - & - & - & - & - & 3.0 & - & - & 2.40 & 7.72 & 0.11 & - & 1111 \\
\hline $8-5,132-135$ (No, 14) & - & 8700 & - & - & - & - & - & 0.2 & 137 & 39 & 107 & 5.7 & - & - & - & - & - & - & - & - & - & - & - \\
\hline $9-1,70-73$ (No. $4 \mathrm{c})$ & - & 8580 & 284 & 265 & 105 & - & - & 0.2 & 137 & 40 & 105 & 6.7 & - & - & - & - & - & - & - & - & - & - & - \\
\hline $9 \cdot 2,135-138(\mathrm{No}, 6)$ & 34 & 8400 & 236 & 300 & 126 & 132 & 138 & 0.3 & 179 & 37 & 108 & 8.2 & 0.04 & 0.08 & 31 & 4.6 & 1.23 & 0.66 & 2.41 & 4.51 & 0.43 & - & 608 \\
\hline $9-3,91-94$ (No. 6c) & 34 & 8280 & - & - & - & 94 & 143 & 2.5 & 173 & 36 & 105 & 8.9 & 0.02 & 0.03 & 31 & 4.3 & 1.31 & 0.64 & 2.43 & 6.74 & 0.39 & - & 926 \\
\hline $9-4,41.45$ (No. 3b) & 33 & 8400 & 241 & 314 & 88 & 96 & 127 & 2.5 & 166 & 34 & 108 & 8.2 & 0.02 & - & 19 & 5.0 & 1.28 & 0.64 & 2.35 & 5.53 & 0.44 & - & 803 \\
\hline $9-5,72-75$ (No. 6$)$ & 34 & 8580 & 241 & 301 & 92 & 102 & 140 & 0.7 & 179 & 32 & 119 & 8.3 & 0.04 & 0.03 & 37 & 5.7 & 1.30 & 0.65 & 2,40 & 5.09 & 0.46 & - & 682 \\
\hline $10-2,19-25$ (No. 3) & 41.6 & 11280 & 389 & 249 & 116 & 120 & 73 & 2.4 & 114 & 47 & 132 & 5.5 & 0.03 & 0.13 & - & 4,4 & 1.71 & 0.96 & 3.42 & 3.67 & 0.21 & - & 590 \\
\hline
\end{tabular}

TABLE 4a

Hole 423 (EPR, $9^{\circ} \mathrm{N}, \sim 1.2$ m.y. old): Major Oxides

\begin{tabular}{|c|c|c|c|c|c|c|c|c|c|c|c|c|}
\hline $\begin{array}{c}\text { Sample } \\
\text { (Interval in } \mathrm{cm} \text { ) }\end{array}$ & $\mathrm{SiO}_{2}$ & $\mathrm{Al}_{2} \mathrm{O}_{3}$ & $\mathrm{Fe}_{2} \mathrm{O}_{3}$ & $\mathrm{MnO}$ & $\mathrm{MgO}$ & $\mathrm{CaO}$ & $\mathrm{Na}_{2} \mathrm{O}$ & $\mathrm{K}_{2} \mathrm{O}$ & $\mathrm{TiO}_{2}$ & $\mathrm{P}_{2} \mathrm{O}_{5}$ & LOI & Total \\
\hline 5 CC, $40-42$ (No. 4$)$ & 50.43 & 13.59 & 11.36 & 0.21 & 6.79 & 11.02 & 2.92 & 0.32 & 2.09 & 0.24 & 1.14 & 100.11 \\
\hline 6-1, 28-34 (No. 5) & 50.14 & 13.40 & 13.02 & 0.20 & 6.92 & 10.97 & 1.94 & 0.23 & 2.06 & 0.23 & 0.83 & 99.94 \\
\hline Section $7-1$, (No. 11) & 49.75 & 13.28 & 13.53 & 0.18 & 6.60 & 10.68 & 2.43 & 0.35 & 2.04 & 0.20 & 1.00 & 100.01 \\
\hline 7-1, 46-47 (No. 7) & 50.37 & 13.55 & 12.70 & 0.18 & 6.88 & 10.75 & - & 0.36 & 2.05 & 0.22 & 0.76 & 97.82 \\
\hline $8-1,40-42$ (No. 6) & 50.90 & 13.55 & 12.31 & 0.18 & 6.82 & 10.87 & 2.57 & 0.35 & 2.10 & 0.22 & 0.81 & 100.67 \\
\hline
\end{tabular}

TABLE 4b

Hole 423 (EPR, $9^{\circ} \mathrm{N}, \sim 1.2$ m.y. old): Trace Elements

\begin{tabular}{|c|c|c|c|c|c|c|c|c|c|c|c|c|c|c|c|c|c|c|c|c|c|c|c|}
\hline $\begin{array}{c}\text { Sample } \\
\text { (Interval in cm) }\end{array}$ & $\begin{array}{l}\mathrm{Sc} \\
\mathrm{NA}\end{array}$ & $\begin{array}{c}\mathrm{Ti} \\
\mathrm{XRF}\end{array}$ & $\begin{array}{l}\mathrm{V} \\
\mathrm{XRF}\end{array}$ & $\underset{\mathrm{XRF}}{\mathrm{Cr}}$ & XRF & NAA & $\begin{array}{c}\mathrm{Ni} \\
\text { NAA }\end{array}$ & $\begin{array}{c}R b \\
\text { XRF }\end{array}$ & $\begin{array}{c}\text { Sr } \\
\text { XRF }\end{array}$ & $\begin{array}{c}\mathrm{Y} \\
\text { XRF }\end{array}$ & $\begin{array}{c}\mathrm{Zr} \\
\text { XRF }\end{array}$ & $\begin{array}{c}\mathrm{Nb} \\
\text { XRF }\end{array}$ & $\begin{array}{l}\mathrm{Sb} \\
\text { NAA }\end{array}$ & $\begin{array}{c}\text { Cs } \\
\text { NAA }\end{array}$ & $\begin{array}{c}\text { Ba } \\
\text { NAA }\end{array}$ & $\begin{array}{c}\mathrm{La} \\
\text { NAA }\end{array}$ & $\begin{array}{c}\text { Eu } \\
\text { NAA }\end{array}$ & $\begin{array}{c}\text { Tb } \\
\text { NAA }\end{array}$ & $\begin{array}{c}\mathrm{Hf} \\
\text { NAA }\end{array}$ & $\begin{array}{c}\mathrm{Ta} \\
\text { NAA }\end{array}$ & $\begin{array}{l}\text { Th } \\
\text { NAA }\end{array}$ & $\underset{\text { NAA }}{U}$ & $\begin{array}{c}\text { W } \\
\text { NAA }\end{array}$ \\
\hline $5 \mathrm{CC}, 40-42(\mathrm{No}, 4)$ & 40.2 & 12540 & 377 & - & 170 & 173 & 100 & - & - & - & - & - & - & 0.10 & - & 4.0 & 2.10 & 1.05 & 3.54 & 8.9 & 0.23 & - & 1308 \\
\hline 6-1, 28-34 (No. 5) & 42 & 12360 & - & - & 104 & 101 & 67 & 1 & 119 & - & 149 & - & - & 0.07 & - & 4.2 & 1.91 & 10.2 & 3.61 & 5.94 & 0.25 & - & - \\
\hline Section 7-1, (No. 1b) & 41.6 & 12240 & 230 & 160 & 97 & 91 & 56 & 4.1 & 131 & 51 & 144 & 10.3 & - & 0.17 & - & 4.0 & 1.58 & 0.96 & 3.49 & 6.27 & 0.19 & - & - \\
\hline $7-1,46-47($ No. 7$)$ & - & 12300 & $\overline{-}$ & - & - & - & - & 2.2 & 118 & 65 & 138.6 & 10.1 & - & - & - & - & - & - & - & - & - & - & - \\
\hline $8-1,40-42($ No, 6$)$ & - & 12600 & 376 & 196 & 146 & - & - & 4.3 & 128 & 61 & 141 & 10 & - & - & - & - & - & - & - & - & - & - & - \\
\hline
\end{tabular}

Figure 5 is a plot of $\mathrm{Zr}$ versus $\mathrm{Ti}$; only slight fractionation is observed between these elements, confirming the hygromagmaphile behavior of $\mathrm{Ti}$ (Bougault, $1977 b$ ). The slope found is $1.1 \times 10^{-2}$, very close to the value obtained at $22^{\circ} \mathrm{N}$ and $36^{\circ} \mathrm{N}$ in the Atlantic: 1.15 $\times 10^{-2}$ (Bougault et al., 1978).

These results confirm that there is little or no fractionation between such pairs of elements as $\mathrm{Y}-\mathrm{Tb}, \mathrm{Zr}$ $\mathrm{Hf}$, and $\mathrm{Nb}-\mathrm{Ta}$. Thus, we conclude that the ratios are distinctive of the mantle prior to differentiation. Since these ratios are the same, or nearly the same, in the Atlantic and Pacific oceans, and in addition are similar to chondritic ratios, it is very probable that they are characteristic of the earth itself.

With respect to two hygromagmaphile elements whose partition coefficients are different by an order of magnitude, for instance $\mathrm{Y}$ and $\mathrm{La}$, it has been shown (Bougault et al., in press 1,2 ) that the $\mathrm{Y} / \mathrm{La}$ ratio may vary widely. Being very similar to the $\mathrm{Sm} / \mathrm{La}$ ratio used by Shilling (1973), it is a function of mantle differentia- 
TABLE 5a

Hole 428 (EPR, $9^{\circ} \mathrm{N}, \sim 2$ m.y. old): Major Oxides

\begin{tabular}{|c|c|c|c|c|c|c|c|c|c|c|c|c|}
\hline $\begin{array}{c}\text { Sample } \\
\text { (Interval in } \mathrm{cm} \text { ) }\end{array}$ & $\mathrm{SiO}_{2}$ & $\mathrm{Al}_{2} \mathrm{O}_{3}$ & $\mathrm{Fe}_{2} \mathrm{O}_{3}$ & $\mathrm{MnO}$ & $\mathrm{MgO}$ & $\mathrm{CaO}$ & $\mathrm{Na}_{2} \mathrm{O}$ & $\mathrm{K}_{2} \mathrm{O}$ & $\mathrm{TiO}_{2}$ & $\mathrm{P}_{2} \mathrm{O}_{5}$ & LOI & Total \\
\hline $5-4,38-40($ No. 4$)$ & 50.62 & 14.40 & 10.62 & 0.18 & 7.25 & 11.97 & 2.30 & 0.14 & 1.45 & 0.14 & 0.45 & 99.52 \\
\hline $5-4,78-80$ (No. 9) & 49.85 & 14.22 & 10.27 & 0.22 & 7.11 & 12.07 & 2.54 & 0.09 & 1.43 & 0.12 & 0.45 & 98.37 \\
\hline $6-2,1-3($ No. 1$)$ & 50.01 & 14.17 & 10.46 & 0.20 & 7.52 & 11.84 & 2.44 & 0.08 & 1.45 & 0.14 & 0.36 & 98.67 \\
\hline
\end{tabular}

TABLE 5b

Hole 428 (EPR, $9^{\circ} \mathrm{N}, \sim 2$ m.y. old): Trace Elements

\begin{tabular}{|c|c|c|c|c|c|c|c|c|c|c|c|c|c|c|c|c|c|c|c|c|c|c|c|}
\hline $\begin{array}{c}\text { Sample } \\
\text { (Interval in cm) }\end{array}$ & $\begin{array}{l}\text { Sc } \\
\text { NA }\end{array}$ & $\underset{\text { XRF }}{\mathrm{Ti}}$ & $\begin{array}{c}\mathrm{V} \\
\mathrm{XRF}\end{array}$ & $\underset{\text { XRF }}{\mathrm{Cr}}$ & XRF & NAA & $\begin{array}{c}\mathrm{Ni} \\
\mathrm{NAA}\end{array}$ & $\begin{array}{c}\mathrm{Rb} \\
\mathrm{XRF}\end{array}$ & $\begin{array}{l}\text { Sr } \\
\text { XRF }\end{array}$ & $\begin{array}{c}\text { Y } \\
\text { XRF }\end{array}$ & $\underset{\mathrm{XRF}}{\mathrm{Zr}}$ & $\begin{array}{c}\mathrm{Nb} \\
\mathrm{XRF}\end{array}$ & $\begin{array}{c}\text { Sb } \\
\text { NAA }\end{array}$ & $\begin{array}{l}\text { Cs } \\
\text { NAA }\end{array}$ & $\underset{\text { NAA }}{\mathrm{Ba}}$ & $\stackrel{\mathrm{La}}{\mathrm{NAA}}$ & $\begin{array}{c}\text { Eu } \\
\text { NAA }\end{array}$ & $\begin{array}{c}\text { Tb } \\
\text { NAA }\end{array}$ & $\begin{array}{c}\text { Hf } \\
\text { NAA }\end{array}$ & $\begin{array}{c}\mathrm{Ta} \\
\text { NAA }\end{array}$ & $\begin{array}{l}\text { Th } \\
\text { NAA }\end{array}$ & $\begin{array}{c}\text { U } \\
\text { NAA }\end{array}$ & $\begin{array}{l}\text { W } \\
\text { NAA }\end{array}$ \\
\hline $5-4,38-40$ (No. 4$)$ & 42.2 & 8700 & 281 & 313 & 41 & 43 & 81 & 1.2 & 139 & 42 & 107 & 5.3 & - & - & - & 3.1 & 1.29 & 0.73 & 2.5 & 0.25 & 0.24 & - & - \\
\hline $5-4,78-80$ (No. 9) & 42.4 & 8580 & 286 & 324 & 41 & 42 & 82 & 0.8 & 128 & 40 & 107 & 5.2 & - & - & 17 & 3.0 & $1.2^{11}$ & 0.72 & 2.44 & 0.24 & 0.21 & - & - \\
\hline $6 \cdot 2,1-3($ No. 1$)$ & 41.9 & 8700 & 298 & 309 & 45 & 42 & 77 & 0.6 & 129 & 40 & 105 & 4.9 & - & - & - & 3.4 & 1.29 & 0.72 & 2.57 & 0.25 & 0.21 & - & - \\
\hline
\end{tabular}

TABLE 6a

Hole 428A (EPR, $9^{\circ} \mathrm{N}, \sim 2 \mathrm{~m} . \mathrm{y}$. old): Major Oxides

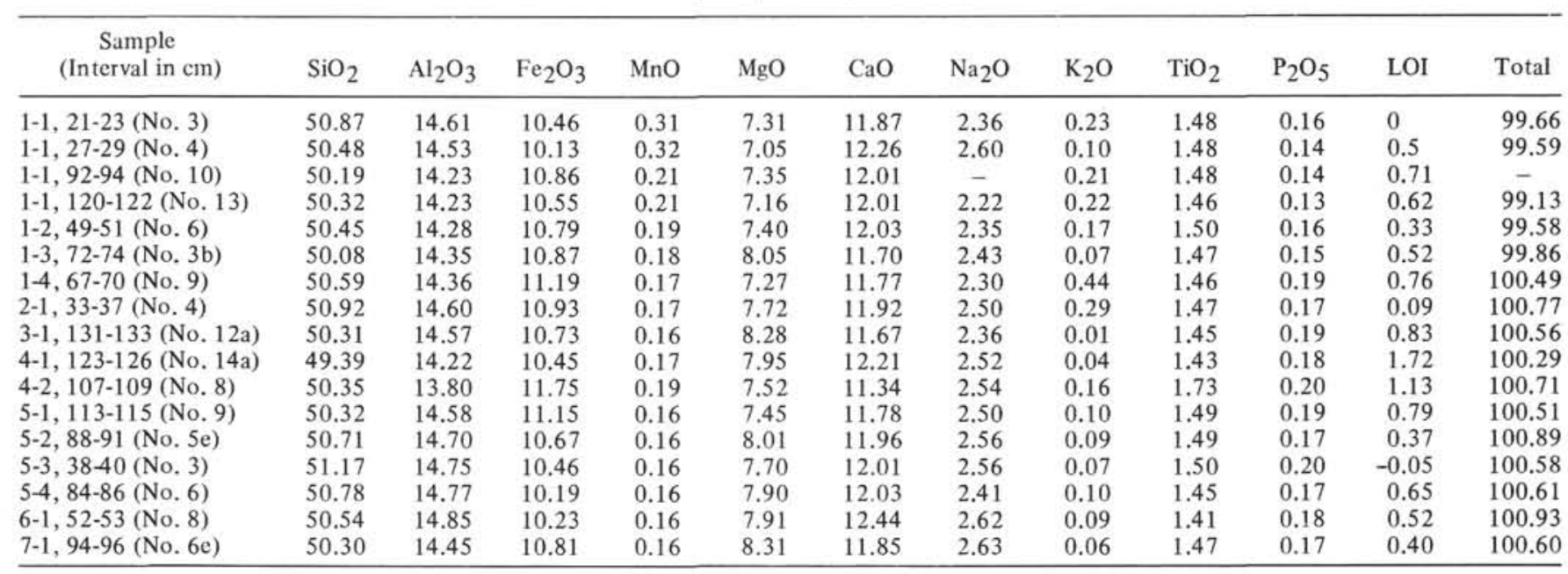

TABLE 6b

Hole 428A (EPR, $9^{\circ} \mathrm{N}, \sim 2$ m.y. old): Trace Elements

\begin{tabular}{|c|c|c|c|c|c|c|c|c|c|c|c|c|c|c|c|c|c|c|c|c|c|c|c|}
\hline $\begin{array}{c}\text { Sample } \\
\text { (Interval in cm) }\end{array}$ & $\begin{array}{l}\mathrm{Se} \\
\mathrm{NA}\end{array}$ & $\begin{array}{c}\mathrm{Ti} \\
\mathrm{XRF}\end{array}$ & $\begin{array}{c}\mathrm{V} \\
\mathrm{XRF}\end{array}$ & $\begin{array}{c}\mathrm{Cr} \\
\mathrm{XRF}\end{array}$ & XRF & NAA & $\underset{\mathrm{NAA}}{\mathrm{Ni}}$ & $\begin{array}{c}\mathrm{Rb} \\
\mathrm{XRF}\end{array}$ & $\begin{array}{c}\text { Sr } \\
\text { XRF }\end{array}$ & $\begin{array}{c}\mathrm{Y} \\
\mathrm{XRF}\end{array}$ & $\begin{array}{c}\mathrm{Zt} \\
\mathrm{XRF}\end{array}$ & $\begin{array}{c}\mathrm{Nb} \\
\mathrm{XRF}\end{array}$ & $\begin{array}{c}\text { Sb } \\
\text { NAA }\end{array}$ & $\begin{array}{l}\text { Cs } \\
\text { NAA }\end{array}$ & $\begin{array}{c}\mathrm{Ba} \\
\mathrm{NAA}\end{array}$ & $\underset{\mathrm{NAA}}{\mathrm{La}}$ & $\begin{array}{c}\text { Eu } \\
\text { NAA }\end{array}$ & $\begin{array}{c}\mathrm{Tb} \\
\text { NAA }\end{array}$ & $\begin{array}{l}\mathrm{Hf} \\
\text { NAA }\end{array}$ & $\begin{array}{c}\mathrm{Ta} \\
\text { NAA }\end{array}$ & $\begin{array}{l}\text { Th } \\
\text { NAA }\end{array}$ & $\begin{array}{c}\text { U } \\
\text { NAA }\end{array}$ & $\begin{array}{l}\text { W } \\
\text { NAA }\end{array}$ \\
\hline $1-1,21-23$ (No. 3) & 41.4 & 8880 & 292 & 320 & 41 & 42 & 83 & - & - & - & - & - & - & 0.05 & 21 & 3.3 & 1.36 & 0.74 & 2.44 & 0.25 & 0.24 & 0.07 & - \\
\hline $1-1,27-29(\mathrm{No}, 4)$ & 42 & 8880 & 299 & 336 & 43 & 43 & 87 & - & 134 & 42 & 110 & 5.0 & - & - & 8.3 & 4.1 & 1.20 & 0.71 & 2.51 & 0.25 & 0.22 & - & - \\
\hline 1-1. $92-94$ (No. 10) & 41.4 & 8880 & - & - & - & 42 & 84 & 3.0 & 131 & - & 101 & 4.5 & - & 0.03 & 13.8 & 3.8 & 1.24 & 0.73 & 2.46 & 0.24 & 0.23 & - & - \\
\hline $1-1,120-122$ (No. 13) & 42.2 & 8760 & 287 & 310 & 40 & 43 & 88 & 2.0 & 135 & 42 & 109 & 5.9 & - & 0.06 & - & 4.0 & 1.25 & 0.72 & 2.45 & 0.26 & 0.23 & 0.12 & - \\
\hline $1-2,49-51$ (No. 6) & 42.3 & 9000 & 293 & 321 & 42 & 42 & 80 & 2.1 & 128 & 40 & 109 & 5.7 & 0.02 & 0.06 & 20.9 & 3. & 1.30 & 0.72 & 2.52 & 0.26 & 0.24 & - & - \\
\hline $1-3.72 .74$ (No. 3b) & 42.6 & 8820 & 300 & 311 & 46 & 42 & 79 & 0.2 & 131 & 38 & 95 & - & 0.03 & 0.01 & 9 & 3.5 & 1.34 & 0.76 & 2.67 & 0.26 & 0.25 & 0.07 & - \\
\hline $14,67.70($ No. 9$)$ & 41.3 & 8760 & 295 & 313 & 48 & 40 & 75 & 6.7 & 129 & 39 & 104 & 5.3 & 0.05 & 0.28 & 15.5 & 3. & 1.30 & 0.71 & 2.43 & 0.25 & 0.22 & 0.13 & - \\
\hline $2-1.33-35($ No. 4$)$ & 42.4 & 8820 & 300 & 309 & 46 & 42 & 82 & 3.9 & 128 & 38 & 101 & 4.9 & 0.05 & 0.17 & - & 3. & 1.24 & 0.75 & 2.42 & 0.25 & 0.26 & - & - \\
\hline 3-1.131-133 (No. 12a) & 41.9 & 8700 & 295 & 316 & 44 & 42 & 84 & 0.6 & 131 & 37 & 92 & 4.6 & 0.03 & 0.03 & - & 3. & 1. & 0. & 2. & 0.2 & 0.22 & - & - \\
\hline $4-1,123-126$ (No. 14a) & 41.8 & 8580 & 291 & 305 & 45 & 42 & 84 & 0.2 & - & 36 & 97 & 4.2 & 0.04 & - & 8.8 & 3. & 1.31 & 0.77 & 2.54 & 0.24 & 0.22 & - & - \\
\hline $4-2,107-109($ No. 8$)$ & 42.4 & 10380 & 290 & 314 & 48 & 42 & 85 & 0.5 & 136 & 39 & 111 & 4.7 & 0.01 & - & 8.7 & 3 . & 1.36 & 0.7 & & 0.2 & 0.26 & 0.13 & - \\
\hline $5-1,113-115$ (No, 9) & 41.6 & 8940 & 304 & 296 & 45 & 42 & 81 & 1.1 & - & 38 & - & 4.4 & 0.0 & 0.05 & - & 3. & 1.25 & 0.72 & 2.78 & 0.26 & 0.22 & - & - \\
\hline $5-2,88-91$ (No. . e ) & 42.1 & 8940 & 302 & 315 & 45 & 42 & 77 & 0.1 & 135 & 38 & - & 4.3 & 0.03 & - & 9 & 3. & 1.30 & & & & & - & - \\
\hline $5-3,38-40(\mathrm{~N})$ & 43.9 & 9000 & 302 & 319 & 46 & 43 & 54 & 0.1 & 131 & 40 & 109 & 5.8 & 0.03 & 0.02 & 22 & 4.4 & 1.41 & 0.87 & 2.51 & 0.28 & 0.23 & 0.09 & - \\
\hline $5-4,84-86(2)$ & 42.3 & 700 & 289 & 375 & 44 & 42 & 87 & 0.1 & 131 & 37 & - & 4.5 & 0.03 & - & 18 & & & & & & 0.20 & 0.11 & - \\
\hline $6-1,52-53($ No. 8$)$ & 41.6 & 460 & 288 & 385 & 45 & 43 & 90 & 0.1 & 134 & 36 & 98 & 4.2 & 0.03 & 0.01 & 11.4 & 3.5 & 1.28 & 0.69 & 2.69 & 0.24 & 0.22 & 0.34 & - \\
\hline $7-1,84-96($ No. $6 \mathrm{c})$ & 42.0 & 8820 & 293 & 315 & 45 & 42 & 83 & 0.8 & 134 & 37 & 97 & 3.3 & - & - & 6 & 4.0 & 1.35 & 0.71 & - & 0.25 & 0.19 & - & - \\
\hline
\end{tabular}

tion and partial melting and can vary within a single hole (Wood et al., in press). For this reason, we believe that this ratio by itself is not sufficient indication of the heterogeneity of different sources in the mantle. Figure 7 shows where the samples of Leg 54 plot in comparison with the data obtained in the North Atlantic. All the Leg
54 samples fall in what has so far been considered to be a "depleted" field. But again, this is not sufficient reason to deduce that these basalts were produced from a "depleted" mantle. A good example is given by some samples of Hole 409 (North Atlantic) which show a "depleted" character (high $\mathrm{Y} / \mathrm{La}$ and $\mathrm{Sm} / \mathrm{La}$ ); but 
TABLE 7a

Hole 429A (EPR, $9^{\circ} \mathrm{N}, \sim 4.5$ m.y. old): Major Oxides

\begin{tabular}{|c|c|c|c|c|c|c|c|c|c|c|c|c|}
\hline $\begin{array}{c}\text { Sample } \\
\text { (Interval in } \mathrm{cm} \text { ) }\end{array}$ & $\mathrm{SiO}_{2}$ & $\mathrm{Al}_{2} \mathrm{O}_{3}$ & $\mathrm{Fe}_{2} \mathrm{O}_{3}$ & $\mathrm{MnO}$ & $\mathrm{MgO}$ & $\mathrm{CaO}$ & $\mathrm{Na}_{2} \mathrm{O}$ & $\mathrm{K}_{2} \mathrm{O}$ & $\mathrm{TiO}_{2}$ & $\mathrm{P}_{2} \mathrm{O}_{5}$ & LOI & Total \\
\hline 1-1, 43-45 (No. 1) & 49.72 & 15.87 & 8.79 & 0.15 & 7.12 & 12.99 & 2.33 & 0.08 & 1.15 & 0.11 & 0.98 & 99.29 \\
\hline $2-1,87-89$ (No. 12) & 50.05 & 13.84 & 12.05 & 0.18 & 6.73 & 11.51 & - & 0.5 & 1.60 & 0.14 & 0.71 & 97.36 \\
\hline Section 3-1, (No. 7b) & 50.57 & 13.89 & 11.91 & 0.16 & 7.07 & 11.44 & 2.42 & 0.08 & 1.61 & 1.15 & 0.43 & 99.73 \\
\hline $2-1,16-18$ (No. 3) & 49.71 & 15.51 & 9.07 & 0.14 & 7.69 & 12.26 & 2.38 & 0.18 & 1.26 & - & 0.99 & 99.19 \\
\hline $2-2,14-16($ No. 2$)$ & 50.23 & 14.14 & 10.58 & 0.18 & 6.73 & 11.69 & 2.57 & 0.31 & 1.47 & - & 0.53 & 98.43 \\
\hline Section 3-1, (No. 1) & 49.23 & 15.75 & 8.65 & 0.15 & 7.05 & 13.18 & 2.41 & 0.06 & 1.20 & - & 1.29 & 98.97 \\
\hline
\end{tabular}

TABLE $7 \mathrm{~b}$

Hole 429A (EPR, $9^{\circ} \mathrm{N}, \sim 4.5$ m.y. old): Trace Elements

\begin{tabular}{|c|c|c|c|c|c|c|c|c|c|c|c|c|c|c|c|c|c|c|c|c|c|c|c|}
\hline $\begin{array}{c}\text { Sample } \\
\text { (Interval in } \mathrm{cm} \text { ) }\end{array}$ & $\begin{array}{l}\text { Sc } \\
\text { NA }\end{array}$ & $\begin{array}{c}\mathrm{Ti} \\
\text { XRF }\end{array}$ & $\begin{array}{c}\mathrm{V} \\
\mathrm{XRF}\end{array}$ & $\begin{array}{l}\mathrm{Cr} \\
\mathrm{XRF}\end{array}$ & XRF & NAA & $\begin{array}{c}\mathrm{Ni} \\
\mathrm{NAA}\end{array}$ & $\begin{array}{l}\mathrm{Rb} \\
\mathrm{XRF}\end{array}$ & $\underset{\mathrm{XRF}}{\mathrm{Sr}}$ & $\stackrel{\mathrm{Y}}{\mathrm{XRF}}$ & $\underset{\mathrm{XRF}}{\mathrm{Zr}}$ & $\underset{\mathrm{XRF}}{\mathrm{Nb}}$ & $\begin{array}{c}\text { Sb } \\
\text { NAA }\end{array}$ & $\begin{array}{c}\text { Cs } \\
\text { NAA }\end{array}$ & $\begin{array}{l}\mathrm{Ba} \\
\text { NAA }\end{array}$ & $\begin{array}{c}\mathrm{La} \\
\mathrm{NAA}\end{array}$ & $\begin{array}{c}\text { Eu } \\
\text { NAA }\end{array}$ & $\begin{array}{l}\mathrm{Tb} \\
\text { NAA }\end{array}$ & $\begin{array}{c}\mathrm{Hf} \\
\text { NAA }\end{array}$ & $\begin{array}{c}\text { Ta } \\
\text { NAA }\end{array}$ & $\begin{array}{l}\text { Th } \\
\text { NAA }\end{array}$ & $\begin{array}{c}\mathrm{U} \\
\text { NAA }\end{array}$ & $\begin{array}{l}\text { W } \\
\text { NAA }\end{array}$ \\
\hline $1,43-45$ (No. 1) & 8.9 & 6900 & 352 & 372 & 52 & 51 & 126 & 0.5 & 140 & 29 & 80 & 3.6 & 0.05 & 0.03 & - & 2.2 & 1.03 & 0.57 & 1.86 & 0.09 & 0.05 & 0.17 & - \\
\hline 2-1, & - & 7560 & 239 & 403 & 46 & - & - & - & - & - & - & - & - & - & - & - & - & - & - & - & - & - & - \\
\hline $2-1,87-89$ (No. 12) & 43.6 & 9960 & - & 157 & 50 & 37 & 47 & 3.7 & 113 & 43 & 107 & 4.0 & 0.02 & 0.49 & 12 & 3.6 & 1.31 & 0.87 & 2.92 & 0.20 & - & 0.15 & - \\
\hline $2-2,14-16$ (s) & 43.2 & 8820 & - & 144 & 40 & 40 & 49 & - & 350 & - & - & - & 0.01 & 0.29 & 26 & 3.8 & 1.4 & 0.8 & 3.0 & 0.21 & 0.14 & 0.09 & - \\
\hline $3-1,6$ & 39 & 720 & - & 398 & 65 & 69 & 203 & 3 & 235 & - & - & - & 0.07 & 0.04 & - & & & & 2. & 0. & 0.0 & 0.15 & - \\
\hline Section 3-1, (No. 7b) & 42.6 & 9660 & 219 & 151 & 49 & 44 & 61 & - & - & - & - & - & - & - & 10 & 3.4 & 1.40 & 0.78 & 2.94 & 0.20 & 0.15 & - & - \\
\hline
\end{tabular}

TABLE 8a

Hole 424 (Galapagos Spreading Center, $0^{\circ} \mathrm{N} 86^{\circ} \mathrm{W}$ ): Major Oxides

\begin{tabular}{|c|c|c|c|c|c|c|c|c|c|c|c|c|}
\hline $\begin{array}{c}\text { Sample } \\
\text { (Interval in } \mathrm{cm} \text { ) }\end{array}$ & $\mathrm{SiO}_{2}$ & $\mathrm{Al}_{2} \mathrm{O}_{3}$ & $\mathrm{Fe}_{2} \mathrm{O}_{3}$ & $\mathrm{MnO}$ & $\mathrm{MgO}$ & $\mathrm{CaO}$ & $\mathrm{Na}_{2} \mathrm{O}$ & $\mathrm{K}_{2} \mathrm{O}$ & $\mathrm{TiO}_{2}$ & $\mathrm{P}_{2} \mathrm{O}_{5}$ & LOI & Total \\
\hline 5-1, 9-14 (No. 2) & 50.33 & 12.70 & 14.99 & 0.21 & 6.51 & 10.56 & 1.99 & 0.15 & 1.89 & 0.19 & 0.62 & 100.13 \\
\hline $5-1,35-40($ No. 5$)$ & 50.15 & 12.70 & 15.06 & 0.21 & 6.23 & 10.56 & 1.97 & 0.14 & 1.87 & 0.18 & 1.14 & 100.19 \\
\hline $5-1,131-136$ (No. 13) & 49.90 & 12.53 & 14.69 & 0.21 & 6.67 & 10.39 & 1.00 & 0.08 & 1.86 & 0.19 & 1.10 & 99.62 \\
\hline $5-2,56-61$ (No. 2b) & 50.31 & 12.84 & 15.27 & 0.20 & 6.71 & 10.43 & 2.78 & 0.05 & 1.89 & 0.16 & 0.18 & 100.81 \\
\hline $5-3,20-23$ (No. 2) & 50.37 & 12.75 & 15.06 & 0.20 & 6.90 & 10.47 & 2.07 & 0.06 & 1.87 & 0.17 & 0.10 & 100.01 \\
\hline $5-4,2-5$ (No. 1$)$ & 50.28 & 12.66 & 15.21 & 0.20 & 6.66 & 10.49 & 2.12 & 0.04 & 1.92 & 0.19 & 0.29 & 100.07 \\
\hline $6-1,32-36$ (No. 5$)$ & 50.30 & 12.64 & 15.20 & 0.20 & 6.71 & 10.39 & 1.94 & 0.01 & 1.89 & 0.18 & 0.29 & 99.76 \\
\hline $6-1,102-107$ (No. 10c) & 50.24 & 12.66 & 15.16 & 0.20 & 6.83 & 10.49 & 2.32 & 0.03 & 1.89 & 0.21 & 0.19 & 100.21 \\
\hline $7-1,0-10$ & 50.59 & 12.85 & 14.63 & 0.21 & 6.77 & 10.79 & 1.86 & 0.16 & 1.86 & 0.19 & 0.43 & 100.35 \\
\hline
\end{tabular}

TABLE $8 b$

Hole 424 (Galapagos Spreading Center, $0^{\circ} \mathrm{N} 86^{\circ} \mathrm{W}$ ): Trace Elements

\begin{tabular}{|c|c|c|c|c|c|c|c|c|c|c|c|c|c|c|c|c|c|c|c|c|c|c|c|}
\hline $\begin{array}{c}\text { Sample } \\
\text { (Interval in } \mathrm{cm} \text { ) }\end{array}$ & $\begin{array}{l}\text { Se } \\
\text { NA }\end{array}$ & $\begin{array}{c}\mathrm{Ti} \\
\text { XRF }\end{array}$ & $\begin{array}{l}\mathrm{V} \\
\text { XRF }\end{array}$ & $\begin{array}{c}\mathrm{Cr} \\
\mathrm{XRF}\end{array}$ & XRF & $\mathrm{NAA}$ & $\begin{array}{c}\mathrm{Ni} \\
\mathrm{NAA}\end{array}$ & $\begin{array}{c}\mathrm{Rb} \\
\mathrm{XRF}\end{array}$ & $\begin{array}{c}\text { St } \\
\text { XRF }\end{array}$ & $\begin{array}{c}\mathrm{Y} \\
\mathrm{XRF}\end{array}$ & $\begin{array}{c}\mathrm{Zt} \\
\mathrm{XRF}\end{array}$ & $\begin{array}{c}\mathrm{Nb} \\
\mathrm{XRF}\end{array}$ & $\begin{array}{l}\text { Sb } \\
\text { NAA }\end{array}$ & $\begin{array}{c}\text { Cs } \\
\text { NAA }\end{array}$ & $\begin{array}{c}\mathrm{Ba} \\
\mathrm{NAA}\end{array}$ & $\begin{array}{c}\mathrm{La} \\
\mathrm{NAA}\end{array}$ & $\begin{array}{c}\text { Eu } \\
\text { NAA }\end{array}$ & $\begin{array}{c}\text { Tb } \\
\text { NAA }\end{array}$ & $\begin{array}{c}\mathrm{Hf} \\
\text { NAA }\end{array}$ & $\begin{array}{l}\mathrm{Ta} \\
\text { NAA }\end{array}$ & $\begin{array}{l}\text { Th } \\
\text { NAA }\end{array}$ & $\begin{array}{l}\text { U } \\
\text { NAA }\end{array}$ & $\begin{array}{l}\text { W } \\
\text { NAA }\end{array}$ \\
\hline $5.1 .9-14$ (No. 2) & 41.5 & 11340 & 439 & 161 & 217 & 199 & 85 & 0.5 & 66 & 53 & 119 & 7.3 & 0.02 & - & - & 2.9 & 1.55 & 0.91 & 2.80 & 5.50 & 0.30 & - & 902 \\
\hline $5-1.35-40(\mathrm{Nos} .5)$ & 43.5 & 11220 & 440 & 76 & 83 & 75 & 58 & 0.5 & 65 & 54 & 119 & 6.6 & - & - & - & 2.6 & 1.59 & 0.99 & 3.05 & 2.31 & 0.19 & 0.32 & 382 \\
\hline $5-1.131 .136(\mathrm{No}, 13)$ & - & 11160 & 433 & 82.7 & 99 & - & - & - & 61 & 53 & 125 & 6.9 & - & - & - & - & - & - & - & - & - & - & - \\
\hline $5 \cdot 2.56-61(\mathrm{No}, 2 \mathrm{~b})$ & 45.5 & 11340 & 456 & 104 & 52 & 84 & 62 & 0.5 & 65 & 55 & 117 & 7.0 & - & - & 17 & 3.1 & 1.63 & 1.00 & 2.86 & 2.90 & 0.19 & - & 410 \\
\hline $5-3,20-23$ (No. 2) & 45.8 & 11220 & 440 & 83 & 94 & 87 & 61 & 0.5 & 73 & 54 & 120 & 7.0 & - & - & - & 3,3 & 1.55 & 1.07 & 3.07 & 2.80 & 0.18 & - & 396 \\
\hline $5-4.2-5(\mathrm{No}, 1)$ & 45.6 & 11520 & 442 & 76 & 92 & 83 & 54 & 0.5 & 64 & 55 & 116 & 7.5 & - & - & - & 3.2 & 1.62 & 1.09 & 2.77 & 3.70 & 0.15 & - & 548 \\
\hline $6-1,32-36($ No. 5$)$ & - & 11340 & 440 & 77 & 84 & - & - & 0.5 & 65 & 55 & 118 & 6.6 & - & - & - & - & - & - & - & - & - & - & - \\
\hline $6-1,102-107(\mathrm{No}, 10 \mathrm{c})$ & 46.4 & 11340 & - & - & - & 92 & 59 & 2.4 & 59 & & 123 & 6.9 & - & - & - & 3.1 & 1.70 & 1.09 & 3.27 & 4.60 & 0.2 & - & 681 \\
\hline $7-1,0-10$ & 46. & 11160 & 444 & 112 & 122 & 120 & 69 & 1.8 & - & 57 & 109 & 5.9 & 0.03 & 0.09 & - & 2.61 & 1.63 & 1.03 & 3.03 & 4.32 & 0.17 & - & 733 \\
\hline
\end{tabular}

when we consider $\mathrm{La} / \mathrm{Ta}$ (discussed below), these basalts are clearly derived from the same "undepleted" source as the other portion of Hole 409 .

Samples from the North Atlantic at $36^{\circ} \mathrm{N}, 45^{\circ} \mathrm{N}$, and $63^{\circ} \mathrm{N}$ all lie on a single line on a La-Ta diagram, the range of variation being 0.2 to $7.8 \mathrm{ppm}$ for Ta, and 1.5 to $50 \mathrm{ppm}$ for $\mathrm{La}$. Seeing that this wide range of concentrations produces no change in the ratio between the two elements signifies that $\mathrm{La}$ does not fractionate with respect to Ta. But for samples from $22^{\circ} \mathrm{N}$ (young crust) and $25^{\circ} \mathrm{N}$ (old crust), a different line is found; Ta ranges from 0.05 to $0.25 \mathrm{ppm}$, and $\mathrm{La}$ from 1.0 to $4.3 \mathrm{ppm}$ (Figure 8). This difference in slope and ratio of La-Ta is attributed to a different property of the mantle between $22^{\circ} \mathrm{N}$ and $25^{\circ} \mathrm{N}$ on the one hand and $36^{\circ} \mathrm{N}, 45^{\circ} \mathrm{N}$, and $63^{\circ} \mathrm{N}$ on the other. It reflects either different values for $\mathrm{La} / \mathrm{Ta}$ in the mantle sources or different partition coefficient ratios for these elements in the two areas. Leg 54 data plot very nearly on the trend defined at $22^{\circ} \mathrm{N}$ to $25^{\circ} \mathrm{N}$ in the Atlantic. They would thus correspond to a mantle "depleted" in lanthanum.

Since the $\mathrm{Y} / \mathrm{Tb}, \mathrm{Zr} / \mathrm{Hf}$, and $\mathrm{Nb} / \mathrm{Ta}$ ratios are constant in both the Atlantic and Pacific oceans, we deduce that these ratios are characteristic of the primordial mantle. If the two fundamental $\mathrm{La} / \mathrm{Ta}$ values which have been found are confirmed by further studies, it may be possible to conclude that sources in the mantle are not completely uniform in composition and that 
TABLE 9a

Hole 424A (Galapagos Spreading Center, $0^{\circ} \mathrm{N} 86^{\circ} \mathrm{W}$ ): Major Oxides

\begin{tabular}{|c|c|c|c|c|c|c|c|c|c|c|c|c|}
\hline $\begin{array}{c}\text { Sample } \\
\text { (Interval in } \mathrm{cm} \text { ) }\end{array}$ & $\mathrm{SiO}_{2}$ & $\mathrm{Al}_{2} \mathrm{O}_{3}$ & $\mathrm{Fe}_{2} \mathrm{O}_{3}$ & $\mathrm{MnO}$ & $\mathrm{MgO}$ & $\mathrm{CaO}$ & $\mathrm{Na}_{2} \mathrm{O}$ & $\mathrm{K}_{2} \mathrm{O}$ & $\mathrm{TiO}_{2}$ & $\mathrm{P}_{2} \mathrm{O}_{5}$ & LOI & Total \\
\hline $4-1,3-5$ (No. 1) & 50.47 & 12.66 & 14.91 & 0.22 & 5.72 & 10.61 & 2.37 & 0.13 & 1.88 & 0.17 & 0.48 & 99.61 \\
\hline
\end{tabular}

asediment: hydrothermal deposit.

TABLE 9b

Hole 424A (Galapagos Spreading Center, $0^{\circ} \mathrm{N} 86^{\circ} \mathrm{W}$ ): Trace Elements

\begin{tabular}{|c|c|c|c|c|c|c|c|c|c|c|c|c|c|c|c|c|c|c|c|c|c|c|c|}
\hline $\begin{array}{c}\text { Sample } \\
\text { (Interval in } \mathrm{cm} \text { ) }\end{array}$ & $\begin{array}{l}\text { Sc } \\
\text { NA }\end{array}$ & $\begin{array}{c}\mathrm{Ti} \\
\text { XRF }\end{array}$ & $\begin{array}{c}V \\
\text { XRF }\end{array}$ & $\begin{array}{c}\mathrm{Cr} \\
\text { XRF }\end{array}$ & XRF & NAA & $\begin{array}{c}\mathrm{Ni} \\
\text { NAA }\end{array}$ & $\begin{array}{c}\mathrm{Rb} \\
\mathrm{XRF}\end{array}$ & $\underset{\mathrm{XRF}}{\mathrm{Sr}}$ & $\stackrel{\mathrm{Y}}{\mathrm{XRF}}$ & $\underset{\mathrm{XRF}}{\mathrm{Zr}}$ & $\begin{array}{c}\mathrm{Nb} \\
\mathrm{XRF}\end{array}$ & $\begin{array}{l}\mathrm{Sb} \\
\text { NAA }\end{array}$ & $\begin{array}{c}\text { Cs } \\
\text { NAA }\end{array}$ & $\begin{array}{c}\mathrm{Ba} \\
\text { NAA }\end{array}$ & $\begin{array}{c}\mathrm{La} \\
\text { NAA }\end{array}$ & $\begin{array}{c}\text { Eu } \\
\text { NAA }\end{array}$ & $\begin{array}{l}\text { Tb } \\
\text { NAA }\end{array}$ & $\begin{array}{c}\mathrm{Hf} \\
\text { NAA }\end{array}$ & $\begin{array}{l}\mathrm{Ta} \\
\text { NAA }\end{array}$ & $\underset{\text { NAA }}{\text { Th }}$ & $\underset{\text { NAA }}{\mathrm{V}}$ & $\stackrel{\text { W }}{\text { NAA }}$ \\
\hline 4-1, 3-5 (No. 1) & 44.3 & - & 439 & 129 & 170 & 167 & 83 & 1 & 63 & 57 & 117 & 7.9 & 0.03 & 0.05 & 17 & 2.9 & 1.84 & 1.03 & 3.04 & 5.85 & 0.18 & - & 1010 \\
\hline $4-1,45-47$ (No. 6$)$ & - & - & 447 & 91 & 114 & - & - & 3 & 62 & 58 & 118 & 7.6 & - & - & - & - & - & - & - & - & - & - & - \\
\hline
\end{tabular}

TABLE 10a

Hole 425B (Galapagos Spreading Center, $0^{\circ} \mathrm{N} 86^{\circ} \mathrm{W}$ ): Major Oxides

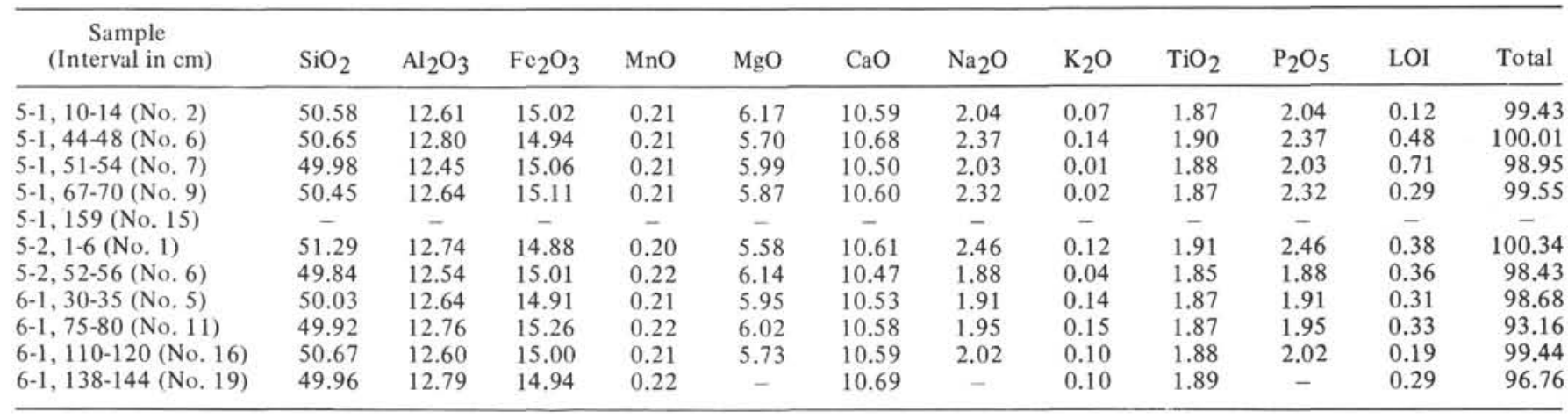

TABLE 10b

Hole 425B (Galapagos Spreading Center, $0^{\circ} \mathrm{N} 86^{\circ} \mathrm{W}$ ): Trace Elements

\begin{tabular}{|c|c|c|c|c|c|c|c|c|c|c|c|c|c|c|c|c|c|c|c|c|c|c|c|}
\hline $\begin{array}{c}\text { Sample } \\
\text { (Interval in cm) }\end{array}$ & $\begin{array}{l}\mathrm{Sc} \\
\mathrm{NA}\end{array}$ & $\begin{array}{c}\mathrm{Ti} \\
\text { XRF }\end{array}$ & $\begin{array}{c}\mathrm{V} \\
\text { XRF }\end{array}$ & $\begin{array}{c}\mathrm{Cr} \\
\mathrm{XRF}\end{array}$ & $\begin{array}{c}{ }^{C} \\
X R F\end{array}$ & NAA & $\begin{array}{l}\mathrm{Ni} \\
\text { NAA }\end{array}$ & $\begin{array}{l}\mathrm{Rb} \\
\mathrm{XRF}\end{array}$ & $\underset{\mathrm{XRF}}{\mathrm{Sr}}$ & $\begin{array}{c}Y \\
X R F\end{array}$ & $\begin{array}{c}\mathrm{Zr} \\
\mathrm{XRF}\end{array}$ & $\begin{array}{c}\mathrm{Nb} \\
\mathrm{XRF}\end{array}$ & $\begin{array}{l}\mathrm{Sb} \\
\mathrm{NAA}\end{array}$ & $\begin{array}{l}\text { Cs } \\
\text { NAA }\end{array}$ & $\begin{array}{l}\mathrm{Ba} \\
\text { NAA }\end{array}$ & $\begin{array}{c}\mathrm{La} \\
\mathrm{NAA}\end{array}$ & $\begin{array}{c}\text { Eu } \\
\text { NAA }\end{array}$ & $\begin{array}{c}\text { Tb } \\
\text { NAA }\end{array}$ & $\begin{array}{c}\mathrm{Hf} \\
\text { NAA }\end{array}$ & $\begin{array}{c}\mathrm{Ta} \\
\mathrm{NAA}\end{array}$ & $\begin{array}{l}\text { Th } \\
\text { NAA }\end{array}$ & $\begin{array}{c}\mathrm{U} \\
\mathrm{NAA}\end{array}$ & $\begin{array}{l}\text { W } \\
\text { NAA }\end{array}$ \\
\hline $5-1,10-14$ (No. 2) & 44.4 & 11220 & 418 & 88 & - & 115 & 60 & 0.1 & 62 & 55 & 112 & 9.5 & - & - & - & 3.1 & 1.73 & 0.99 & 3.17 & 9.02 & 0.24 & - & 1336 \\
\hline 5-1, 44-48 (No. 6) & - & 11400 & 443 & 111 & $=$ & - & - & 1.9 & 62 & 55 & 114 & 8.2 & - & - & - & - & - & - & - & - & - & - & - \\
\hline $5-1,51-54$ (No. 7 ) & 45.4 & 11280 & 444 & 83 & 197 & 92 & 63 & 0.1 & 63 & 50 & 108 & 9.1 & 0.02 & 0.03 & - & 3.1 & 1.57 & 1.02 & 3.16 & 3.9 & 0.23 & - & 567 \\
\hline $5-1,67-70$ (No. 9) & 44.7 & 11220 & 438 & 103 & 81 & 196 & 63 & 0.1 & 63 & 55 & 116 & 10.3 & - & - & - & 3.1 & 1.5 & 0.8 & 3.0 & 9.8 & 0.24 & - & 1552 \\
\hline $5-2,1-6($ No. 1$)$ & 45.4 & 11460 & 445 & 84 & 89 & 75 & 57 & 1.5 & 65 & 57 & 115 & 7.3 & 0.04 & 0.04 & - & 3.1 & 1.63 & 1.02 & 3.19 & 2.54 & 0.22 & 0.26 & 350 \\
\hline $5-2,52-56$ (No. 6 ) & - & 11100 & 424 & 80 & 111 & - & - & 0.1 & 63 & 52 & 117 & 8.2 & - & - & - & - & - & - & - & - & - & - & - \\
\hline $6-1,30-35$ (No. 5 ) & 46.3 & 11220 & 442 & 78 & - & 107 & 58 & 0.4 & 63 & 54 & 113 & 9.3 & 0.02 & - & - & 2.9 & 1.63 & 1.01 & 2.99 & 7.52 & 0.22 & - & 1157 \\
\hline $6-1,75-80($ No. 11$)$ & 45.5 & 11220 & - & 94 & 106 & 56 & 66 & 1.7 & 62 & 54 & 114 & 7.9 & 0.02 & 0.07 & - & 3.1 & 1.66 & 1.03 & 3.12 & 7.9 & 0.14 & - & 1197 \\
\hline $6-1,110-120$ (No. 16) & - & 11280 & 428 & - & - & 101 & 62 & 0.1 & 61 & - & 92 & - & - & 0.02 & - & - & 1.68 & 1.09 & 3.25 & 6.5 & 0.20 & - & 1005 \\
\hline
\end{tabular}

they are not differentiated from the primordial mantle in the same way. The different values found for such ratios as $\mathrm{Y} / \mathrm{La}$ and $\mathrm{Sm} / \mathrm{La}$ are a consequence of both heterogeneous mantle sources and variable partial melting processes.

\section{CONCLUSION}

Grinding samples in a tungsten carbide mortar introduces considerable contamination of $\mathrm{Co}$ and $\mathrm{Ta}$, and, to a lesser extent, $\mathrm{Nb}$.

$\mathrm{Y} / \mathrm{Tb}, \mathrm{Zr} / \mathrm{Hf}$, and $\mathrm{Nb} / \mathrm{Ta}$ ratios have the same single values close to chondrites as in the North Atlantic and are probably characteristic of a primordial mantle. The $\mathrm{La} / \mathrm{Ta}$ ratio, in agreement with the $\mathrm{Y} / \mathrm{La}$ ratio, shows that Leg 54 basalts derive from a so-called depleted mantle. The $\mathrm{La} / \mathrm{Ta}$ ratio is close to the value obtained at $22^{\circ} \mathrm{N}$ and $25^{\circ} \mathrm{N}$ in the Atlantic but different from that at $36^{\circ} \mathrm{N}, 45^{\circ} \mathrm{N}$, and $63^{\circ} \mathrm{N}$ in the North Atlantic ("undepleted" mantle).

\section{REFERENCES}

Bougault H., 1977a. Major elements: analytical chemistry on board and preliminary results. In Aumento, F., Melson, W. G., et al., Initial Reports of the Deep Sea Drilling Project, v. 37: Washington (U.S. Government Printing Office), p. 643-652.

1977b. First transition series elements: fractional crystallization and partial melting. In Aumento, F., Melson, W. G., et al., Initial Reports of the Deep Sea Drilling Proj- 
TABLE 11a

Hole 424C (Galapagos Spreading Center, $0^{\circ} \mathrm{N} 86^{\circ} \mathrm{W}$ ): Major Oxides

\begin{tabular}{|c|c|c|c|c|c|c|c|c|c|c|c|c|}
\hline $\begin{array}{c}\text { Sample } \\
\text { (Interval in } \mathrm{cm} \text { ) }\end{array}$ & $\mathrm{SiO}_{2}$ & $\mathrm{Al}_{2} \mathrm{O}_{3}$ & $\mathrm{Fe}_{2} \mathrm{O}_{3}$ & $\mathrm{MnO}$ & $\mathrm{MgO}$ & $\mathrm{CaO}$ & $\mathrm{Na}_{2} \mathrm{O}$ & $\mathrm{K}_{2} \mathrm{O}$ & $\mathrm{TiO}_{2}$ & $\mathrm{P}_{2} \mathrm{O}_{5}$ & LOI & Total \\
\hline 2-1, 8-15 (No. 2) & 50.69 & 12.74 & 15.17 & 0.22 & 6.09 & 10.52 & 2.26 & 0.06 & 1.86 & 0.17 & 0.33 & 100.11 \\
\hline $3-1,16-31$ (No. 2) & 50.72 & 12.70 & 15.27 & 0.21 & 6.23 & 10.61 & 2.28 & 0.03 & 1.88 & 0.15 & 0 & 100.08 \\
\hline $3-1,44-57$ (No. 4) & 50.92 & 12.76 & 15.05 & 0.22 & 5.89 & 10.65 & 2.37 & 0.14 & 1.88 & 0.15 & 0.19 & 100.21 \\
\hline
\end{tabular}

TABLE 11b

Hole $424 \mathrm{C}$ (Galapagos Spreading Center, $0^{\circ} \mathrm{N} 86^{\circ} \mathrm{W}$ ): Trace Elements

\begin{tabular}{|c|c|c|c|c|c|c|c|c|c|c|c|c|c|c|c|c|c|c|c|c|c|c|c|}
\hline $\begin{array}{c}\text { Sample } \\
\text { (Interval in } \mathrm{cm} \text { ) }\end{array}$ & $\begin{array}{l}\mathrm{Sc} \\
\mathrm{NA}\end{array}$ & $\underset{\mathrm{XRF}}{\mathrm{Ti}}$ & $\underset{X R F}{V}$ & $\begin{array}{c}\mathrm{Cr} \\
\mathrm{XRF}\end{array}$ & ${ }_{\mathrm{XRF}}^{\mathrm{C}}$ & NAA & $\begin{array}{c}\mathrm{Ni} \\
\text { NAA }\end{array}$ & $\begin{array}{c}\mathrm{Rb} \\
\mathrm{XRF}\end{array}$ & $\begin{array}{c}\mathrm{Sr} \\
\text { XRF }\end{array}$ & $\begin{array}{c}\mathrm{Y} \\
\mathrm{XRF}\end{array}$ & $\begin{array}{c}\mathrm{Zr}_{\mathrm{r}} \\
\mathrm{XRF}\end{array}$ & $\begin{array}{c}\mathrm{Nb} \\
\mathrm{XRF}\end{array}$ & $\begin{array}{c}\mathrm{Sb} \\
\text { NAA }\end{array}$ & $\begin{array}{c}\mathrm{Cs}_{5} \\
\text { NAA }\end{array}$ & $\begin{array}{c}\text { Ba } \\
\text { NAA }\end{array}$ & $\begin{array}{c}\mathrm{La} \\
\text { NAA }\end{array}$ & $\begin{array}{c}\text { Eu } \\
\text { NAA }\end{array}$ & $\begin{array}{c}\mathrm{Tb} \\
\text { NAA }\end{array}$ & $\underset{\text { NAA }}{\mathrm{Hf}}$ & $\begin{array}{c}\mathrm{Ta} \\
\text { NAA }\end{array}$ & $\begin{array}{c}\text { Th } \\
\text { NAA }\end{array}$ & $\underset{\text { NAA }}{U}$ & $\begin{array}{l}\text { W } \\
\text { NAA }\end{array}$ \\
\hline $2-1,8-15$ (No. 2) & 44.2 & 11160 & 421 & 86 & 136 & 132 & 56 & 0.5 & 64 & 53 & 123 & 8.5 & - & - & - & 2.7 & 1.41 & 0.96 & 3.20 & 11.92 & 0.17 & - & 1773 \\
\hline Section $2-1$, (No. 7 ) & 45.9 & 11220 & 442 & 98 & 206 & 211 & 64 & 0.7 & 66 & - & 113 & 9.4 & - & - & - & 3.2 & 1.67 & 1.20 & 3.26 & 8.45 & 0.20 & 2.1 & 1207 \\
\hline $3-1,16-31$ (No. 2) & 45.8 & 11280 & 438 & 69 & 89 & 85 & 49 & 0.5 & 66 & 52 & 122 & 7.8 & 0.01 & - & - & 3. & 1.4 & 1. & 3.2 & 5.20 & 0.17 & - & 755 \\
\hline 3-1, 44-57 (No. 4) & 45,1 & 11280 & 427 & 102 & 91 & 86 & 51 & 0.5 & 66 & 54 & 126 & 6.1 & - & 0.03 & - & 3.1 & 1.40 & 1.06 & 3.3 & 4.68 & 0.20 & - & 713 \\
\hline
\end{tabular}

TABLE 12a

Hole 425 (Galapagos Spreading Center, $60 \mathrm{~km}$ from the axis): Major Oxides

\begin{tabular}{|c|c|c|c|c|c|c|c|c|c|c|c|c|}
\hline $\begin{array}{c}\text { Sample } \\
\text { (Interval in } \mathrm{cm} \text { ) }\end{array}$ & $\mathrm{SiO}_{2}$ & $\mathrm{Al}_{2} \mathrm{O}_{3}$ & $\mathrm{Fe}_{2} \mathrm{O}_{3}$ & $\mathrm{MnO}$ & $\mathrm{MgO}$ & $\mathrm{CaO}$ & $\mathrm{Na}_{2} \mathrm{O}$ & $\mathrm{K}_{2} \mathrm{O}$ & $\mathrm{TiO}_{2}$ & $\mathrm{P}_{2} \mathrm{O}_{5}$ & LOI & Total \\
\hline 7-1, 44-47 (No. 7) & 50.44 & 13.55 & 12.92 & 0.18 & 7.03 & 11.28 & 2.18 & 0.02 & 1.42 & 0.09 & 0.10 & 99.20 \\
\hline 7-2, 4-6 (No. 1) & - & - & - & - & - & - & 2.05 & - & - & - & - & - \\
\hline 7-2, 52-54 (No. 7a) & 50.58 & 14.06 & 11.63 & 0.17 & 7.58 & 11.94 & 2.08 & 0.01 & 1.19 & 0.07 & 0.01 & 99.32 \\
\hline 7-2, 55-59 (No. 7a) & 50.89 & 13.55 & 12.75 & 0.14 & 6.90 & 11.64 & 2.10 & 0.14 & 1.36 & 0.11 & 0.29 & 99.86 \\
\hline $7-2,90-95$ (No. 8b) & 50.56 & 13.71 & 11.95 & 0.18 & 7.48 & 11.88 & 2.07 & 0.015 & 1.17 & 0.10 & 0.33 & 99.43 \\
\hline $9-1,20-28$ (No. 5$)$ & 50.61 & 13.77 & 11.99 & 0.17 & 7.51 & 11.69 & 2.05 & 0.03 & 1.18 & 0.08 & 0.24 & 99.31 \\
\hline 9-1, 59-66 (No. 9) & 50.32 & 13.95 & 11.57 & 0.19 & 7.59 & 12.30 & 2.08 & 0.08 & 0.99 & 0.08 & 0.43 & 99.57 \\
\hline $9-1,106-110$ (No. 15) & 50.14 & 13.99 & 11.53 & 0.18 & 7.33 & 12.32 & 1.96 & 0.08 & 0.98 & 0.06 & 0.76 & 99.32 \\
\hline $9-2,68-73$ (No. 7) & 50.44 & 13.72 & 11.58 & 0.17 & 7.65 & 12.05 & 1.98 & 0.03 & 0.97 & 0.09 & 0.62 & 99.30 \\
\hline 9-3, 3-5 (No. 1) & 50.49 & 13.88 & 11.53 & 0.17 & 7.71 & 11.94 & 1.83 & 0.05 & 0.99 & 0.06 & 0.33 & 98.98 \\
\hline $9-3,62-65$ (No. 9) & 51.48 & 14.33 & 10.78 & 0.17 & 7.28 & 12.47 & 2.04 & 0.01 & 0.99 & 0.08 & -0.33 & 99.58 \\
\hline
\end{tabular}

TABLE 12b

Hole 425 (Galapagos Spreading Center, $60 \mathrm{~km}$ from the axis): Trace Elements

\begin{tabular}{|c|c|c|c|c|c|c|c|c|c|c|c|c|c|c|c|c|c|c|c|c|c|c|c|}
\hline $\begin{array}{l}\text { Sample } \\
\text { (Interval in } \mathrm{cm} \text { ) }\end{array}$ & $\begin{array}{r}\text { Sc } \\
\text { NA }\end{array}$ & $\begin{array}{c}\mathrm{Ti} \\
\mathrm{XRF}\end{array}$ & $\begin{array}{c}V \\
\text { XRF }\end{array}$ & $\begin{array}{l}\mathrm{Cr}_{\mathrm{r}} \\
\mathrm{XRF}\end{array}$ & $\begin{array}{r}C \\
X R F\end{array}$ & NAA & $\begin{array}{l}\mathrm{Ni} \\
\mathrm{NAA}\end{array}$ & $\begin{array}{l}\mathrm{Rb} \\
\mathrm{XRF}\end{array}$ & $\begin{array}{c}\text { Sr } \\
\text { XRF }\end{array}$ & $\begin{array}{c}\mathrm{Y} \\
\text { XRF }\end{array}$ & $\begin{array}{c}\mathrm{Zr} \\
\mathrm{XRF}\end{array}$ & $\begin{array}{l}\mathrm{Nb} \\
\text { XRF }\end{array}$ & $\begin{array}{c}\mathrm{Sb} \\
\text { NAA }\end{array}$ & $\begin{array}{l}\text { Cs } \\
\text { NAA }\end{array}$ & $\begin{array}{l}\mathrm{Ba} \\
\text { NAA }\end{array}$ & $\begin{array}{l}\mathrm{La} \\
\text { NAA }\end{array}$ & $\begin{array}{c}\text { Eu } \\
\text { NAA }\end{array}$ & $\begin{array}{c}\text { Tb } \\
\text { NAA }\end{array}$ & $\begin{array}{l}\text { Hf } \\
\text { NAA }\end{array}$ & $\begin{array}{l}\text { Ta } \\
\text { NAA }\end{array}$ & $\begin{array}{l}\text { Th } \\
\text { NAA }\end{array}$ & $\underset{\text { NAA }}{U}$ & $\begin{array}{l}\text { W } \\
\text { NAA }\end{array}$ \\
\hline 7-1, 44-47 (No. 7) & - & 8520 & 375 & 141 & 107 & $\overline{-}$ & $=$ & 0.1 & 56 & 46 & 68 & 6.3 & - & - & - & - & - & - & $\overline{0}$ & - & - & - & - \\
\hline $7-1,81-85($ No. 11$)$ & 42.5 & - & - & - & - & 389 & 75 & - & - & - & - & - & - & - & - & 1.25 & 0.87 & 0.74 & 2.2 & 7.5 & - & - & 1240 \\
\hline 7-1, 4-6 (No. 1) & 41.7 & - & - & - & - & 84 & 83 & - & - & - & - & - & - & 0.02 & - & 1.12 & 0.91 & 0.61 & 1.71 & 4.25 & 0.01 & - & 640 \\
\hline 7-2,52-54 (No. 7a) & 41.8 & 7140 & 323 & 215 & 141 & 91 & 80 & 0.1 & 57 & 36 & 67 & 5.6 & - & - & - & 1.30 & 1.11 & 0.63 & 1.84 & 4.39 & - & - & 709 \\
\hline 7-2, 90-95 (No. 8b) & - & 7020 & 331 & 227 & 133 & - & $\overline{0}$ & 0.1 & 56 & 34 & 65 & 4.0 & - & - & - & - & - & - & - & - & - & - & - \\
\hline $8-1,105-110$ (No. 12) & 39.3 & 5880 & 286 & 311 & 90 & 94 & 85 & 0.1 & 54 & - & - & 1.5 & - & - & - & 1.04 & 0.84 & 0.53 & 1.47 & 4.67 & - & - & 653 \\
\hline $9-1,20-28$ (No. 5 ) & - & 7080 & 327 & 241 & 101 & - & - & 0.1 & 53 & 35 & 62 & 4.0 & - & - & - & - & - & - & - & - & - & - & - \\
\hline $9-1,59-66$ (No. 9) & - & 5940 & 319 & 263 & 115 & - & - & 0.1 & 49 & 34 & 50 & 4.1 & - & - & - & - & - & - & - & - & - & - & - \\
\hline $9-1,106-110$ (No. 15) & 41.5 & 5880 & 317 & 300 & 90 & 91 & 104 & 0.1 & 47 & 35 & 51 & 4.2 & 0.05 & 0.06 & - & 0.92 & 0.90 & 0.53 & 1.28 & 3.69 & 0.04 & - & 615 \\
\hline 9-2, 68-73 (№. 7) & 41.8 & 5820 & 475 & 264 & 120 & 123 & 91 & 0.5 & 48 & 30 & 55 & 3.7 & - & - & - & 0.81 & 0.64 & 0.55 & 1.5 & 4.21 & 0.01 & - & 734 \\
\hline 9-3, 3-5 (No. 1) & - & 5940 & 309 & 244 & 135 & - & - & 0.1 & - & 33 & 53 & 7.4 & - & - & - & - & - & - & - & - & - & - & $\overline{-}$ \\
\hline 9-3, 68-65 (No. 9) & 42.6 & 5940 & 318 & 294 & 99 & 101 & 106 & - & 50 & 31 & 47 & 4.4 & 0.02 & - & - & 0.77 & 0.89 & 0.57 & 1.49 & 5.45 & - & - & 790 \\
\hline
\end{tabular}

ect, v. 37: Washington (U.S. Government Printing Office), p. 539-546.

Bougault H., Treuil M., and Joron J. L., 1978. Trace elements in basalts from $22^{\circ} \mathrm{N}$ and $36^{\circ} \mathrm{N}$ in the Atlantic ocean: fractional crystallization, partial melting, and heterogeneity of the upper mantle. In Melson, W. G., Rabinowitz, P. D., et al., Initial Reports of the Deep Sea Drilling Project, v. 45: Washington (U.S. Government Printing Office), p. 493506.

Bougault H., Cambon P., Corre O., Joron J. L., and Treuil M., in press. Evidence for variability of magmatic processes and upper mantle heterogeneity in the axial region of the mid-atlantic ridge near $22^{\circ} \mathrm{N}$ and $36^{\circ} \mathrm{N}$. Tectonophysics.

Bougault H., Joron J. L., Treuil M., in press. Alteration, fractional crystallization, partial melting, mantle properties from trace elements data recovered in the North Atlantic. 2nd Ewing Symposium, Lamont-Doherty Geological Observatory, 1978.

Joron J. L., Bollinger C., Quisefit J. P., Bougault H., and Treuil M., in press. Trace elements in basalts at $25^{\circ} \mathrm{N}$ old crust, in the Atlantic Ocean: Alteration, mantle and magmatic processes. In Donnelly, T., Francheteau, J., Bryan, 
TABLE 13 a

Hole 427 (Siqueiros fracture zone, $8^{\circ} \mathrm{N} \sim 5 \mathrm{~m} . \mathrm{y}$. old): Major Oxides

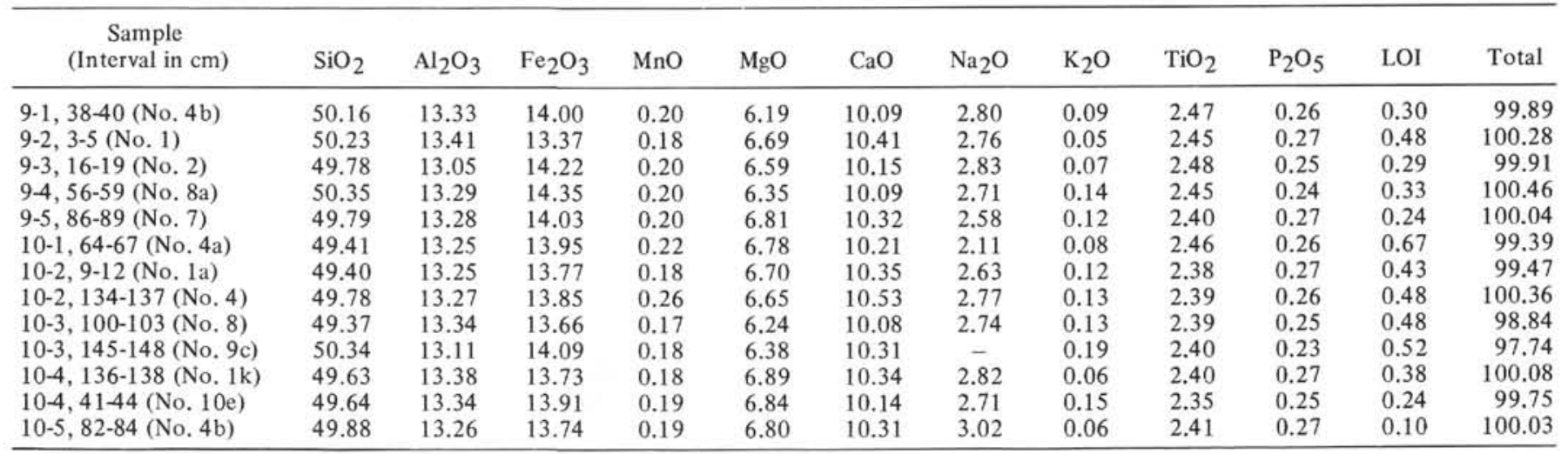

TABLE $13 b$

Hole 427 (Siqueiros fracture zone, $8^{\circ} \mathrm{N} \sim 5$ m.y. old): Trace Elements

\begin{tabular}{|c|c|c|c|c|c|c|c|c|c|c|c|c|c|c|c|c|c|c|c|c|c|c|c|}
\hline $\begin{array}{c}\text { Sample } \\
\text { (Interval in } \mathrm{cm} \text { ) }\end{array}$ & $\begin{array}{l}\text { Sc } \\
\text { NA }\end{array}$ & $\underset{\mathrm{XRI}}{\mathrm{Ti}}$ & $\underset{X R 1}{V}$ & $\begin{array}{c}\mathrm{Cr} \\
\mathrm{XRF}\end{array}$ & $\begin{array}{r}\mathrm{XRF} \\
\end{array}$ & NAA & $\underset{\mathrm{NAA}}{\mathrm{Ni}}$ & $\begin{array}{l}\mathrm{Rb} \\
\mathrm{XRI}\end{array}$ & $\underset{\mathrm{XRF}}{\mathrm{Sr}}$ & $\begin{array}{c}\mathrm{Y} \\
\mathrm{XRF}\end{array}$ & $\begin{array}{l}\mathrm{Zt} \\
\text { XRF }\end{array}$ & $\begin{array}{l}\mathrm{Nb} \\
\mathrm{XRF}\end{array}$ & $\stackrel{\mathrm{Sb}}{\mathrm{NAA}}$ & $\begin{array}{c}\mathrm{CS}_{\mathrm{S}} \\
\mathrm{NAA}\end{array}$ & $\begin{array}{c}\mathrm{Ba} \\
\text { NAA }\end{array}$ & $\underset{\text { NAA }}{\text { La }}$ & $\begin{array}{c}\text { Eu } \\
\text { NAA }\end{array}$ & $\begin{array}{c}\text { Tb } \\
\text { NAA }\end{array}$ & $\begin{array}{c}\mathrm{Hf} \\
\mathrm{NAA}\end{array}$ & $\begin{array}{c}\mathrm{Ta} \\
\text { NAA }\end{array}$ & $\begin{array}{c}\text { Th } \\
\text { NAA }\end{array}$ & $\underset{\text { NAA }}{U}$ & $\begin{array}{c}\text { W } \\
\text { NAA }\end{array}$ \\
\hline $9 \cdot 1,38-40(N o, 4 b)$ & - & 14820 & 415 & 111 & 46 & & - & 0.9 & 127 & 70 & 187 & 8.4 & _- & - & & - & - & - & - & - & - & - & - \\
\hline $9 \cdot 2,3-5$ (No. 1$)$ & 44.6 & 14700 & 423 & 112 & 49 & 43 & 61 & - & - & & - & - & 0.03 & - & - & 6.0 & 1.94 & 1.24 & 4.48 & 0.38 & 0.28 & - & - \\
\hline $9-3,16.19($ No. 2$)$ & 44.3 & 14880 & 426 & 156 & - & 44 & 55 & - & & 64 & 173 & 7.1 & nos & - & - & 6.3 & 1.86 & 1.14 & 4.56 & 0.37 & 0.33 & - & - \\
\hline $9-4,56-59$ (No. 8a) & 43.7 & 14700 & 431 & 114 & 51 & 44 & 57 & 0.6 & 124 & 70 & 178 & 7.2 & - & - & - & 6.0 & 1.84 & 1.20 & 4.52 & 0.36 & 0.31 & - & - \\
\hline $9-5,86-89(\mathrm{No}, 7)$ & 45 & 14400 & 419 & iii & 49 & 44 & 61 & 0.7 & 125 & 67 & 175 & 7.1 & - & 0.01 & 24 & 6.2 & 1.88 & 1.20 & 4.46 & 0.37 & 0.31 & 0.17 & - \\
\hline $10-1,64-67(\mathrm{No}, 4 \mathrm{a})$ & 43.5 & 14760 & 418 & 97 & 87 & - & 77 & 0.1 & 121 & 67 & 177 & 9.8 & 0.03 & - & $=$ & 5.6 & 1.98 & 1.21 & 4.21 & 4.21 & 0.31 & 0.35 & 717 \\
\hline $0-2,9-12$ (No, 1a) & - & 14280 & 394 & 146 & 79 & - & & 0.5 & & 62 & 175 & 7.1 & - & - & - & - & - & - & - & - & - & - & - \\
\hline $10-2,134-137\left(N_{0}, 4\right)$ & 44 & 14340 & 416 & 123 & 49 & 44 & 61 & - & 126 & 68 & 178 & 7.6 & 0.03 & - & - & 5.5 & 1.86 & 1.19 & 4.22 & 0.36 & 0.30 & - & - \\
\hline $10-3,100-103($ No, 8$)$ & 43 & 14340 & 415 & 132 & 80 & 74 & 66 & 0.8 & 121 & 65 & 178 & 9.2 & 0.01 & - & 28 & 5.7 & 1.96 & 1.18 & 4.25 & 3,39 & 0.33 & - & 522 \\
\hline $10-3,145-148($ No, $9 \mathrm{c})$ & - & 14400 & 418 & 114 & 50 & - & - & 0.4 & & 69 & 187 & 7.5 & - & - & & - & - & - & - & - & - & - & - \\
\hline $10-4,136-138(\mathrm{No}, 1 \mathrm{k})$ & & 14400 & 421 & 116 & 49 & - & & 0.4 & 122 & 69 & 176 & 7.2 & - & - & - & - & - & - & - & - & - & - & - \\
\hline $10-4.41 .44($ No. $10 \mathrm{c})$ & 43 & 14100 & 413 & 120 & 81 & 78 & 64 & 0.8 & 118 & 66 & 168 & 8.9 & 0.02 & 0.03 & - & 5.9 & 1.79 & 1.09 & 4.08 & 3.62 & 0.30 & - & 498 \\
\hline $10-5.88-84$ (No. $4 b)$ & 43 & 14460 & 415 & 104 & 102 & 97 & 82 & & 117 & 66 & 173 & 9.7 & 0.02 & & - & 5.7 & 1.96 & 1.16 & 4.26 & 5.51 & 0.33 & - & 779 \\
\hline
\end{tabular}

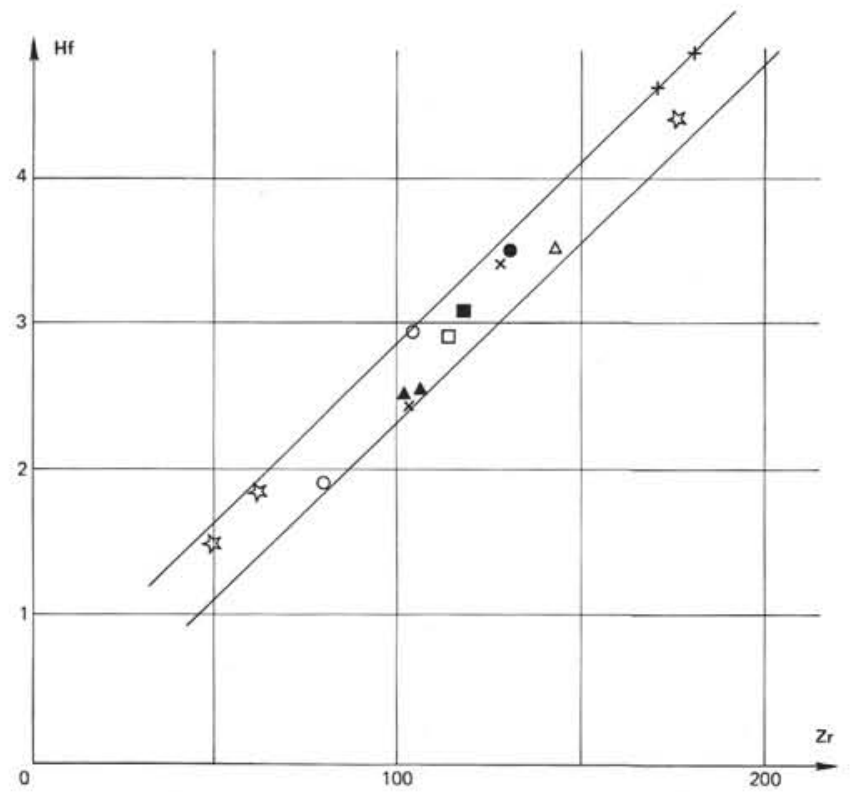

Figure 3. Hf (ppm) versus $\mathrm{Zr}$ (ppm) : the two parallel lines define the range where the North Atlantic data plot. Symbols as for Figure 2.

W., Robinson, P., Flower, M., Salisbury, M., et al., Initial Reports of the Deep Sea Drilling Project, v. 51, 52, 53, Part 2: Washington (U.S. Government Printing Office).

Schilling, J. G., 1973. Icelandic mantle plume: geochemical study of Reykjanes Ridge, Nature, v. 242, p. 565-571.

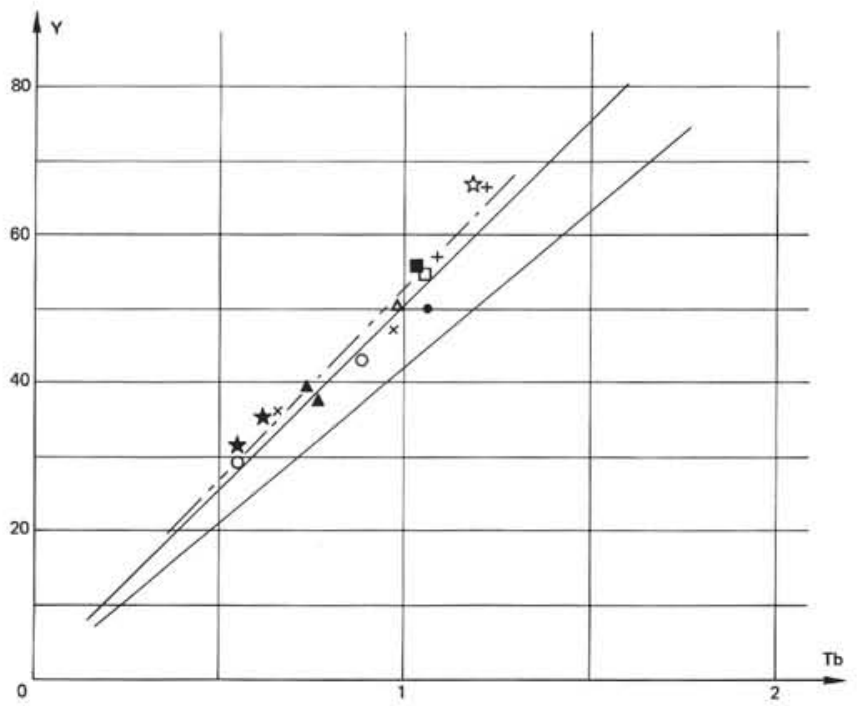

Figure 4. $Y(\mathrm{ppm})$ versus $T b(\mathrm{ppm})$ : the two lines define the range where the North Atlantic data plot. Symbols as for Figure 2.

Wood D. A., Varet J., Bougault H., Corre O., Joron J. L., Treuil M., Bizouard H., Norry M. J., Hawkesworth C. J., and Roddick J. C., 1979. The petrology, geochemistry and mineralogy of North Atlantic basalts: a discussion based on IPOD Leg 49. In Luyendyk, B. P., Cann, J. R., et. al.,Initial Reports of the Deep Sea Drilling Project, v. 49: Washington (U.S. Government Printing Office), p. 597655 . 

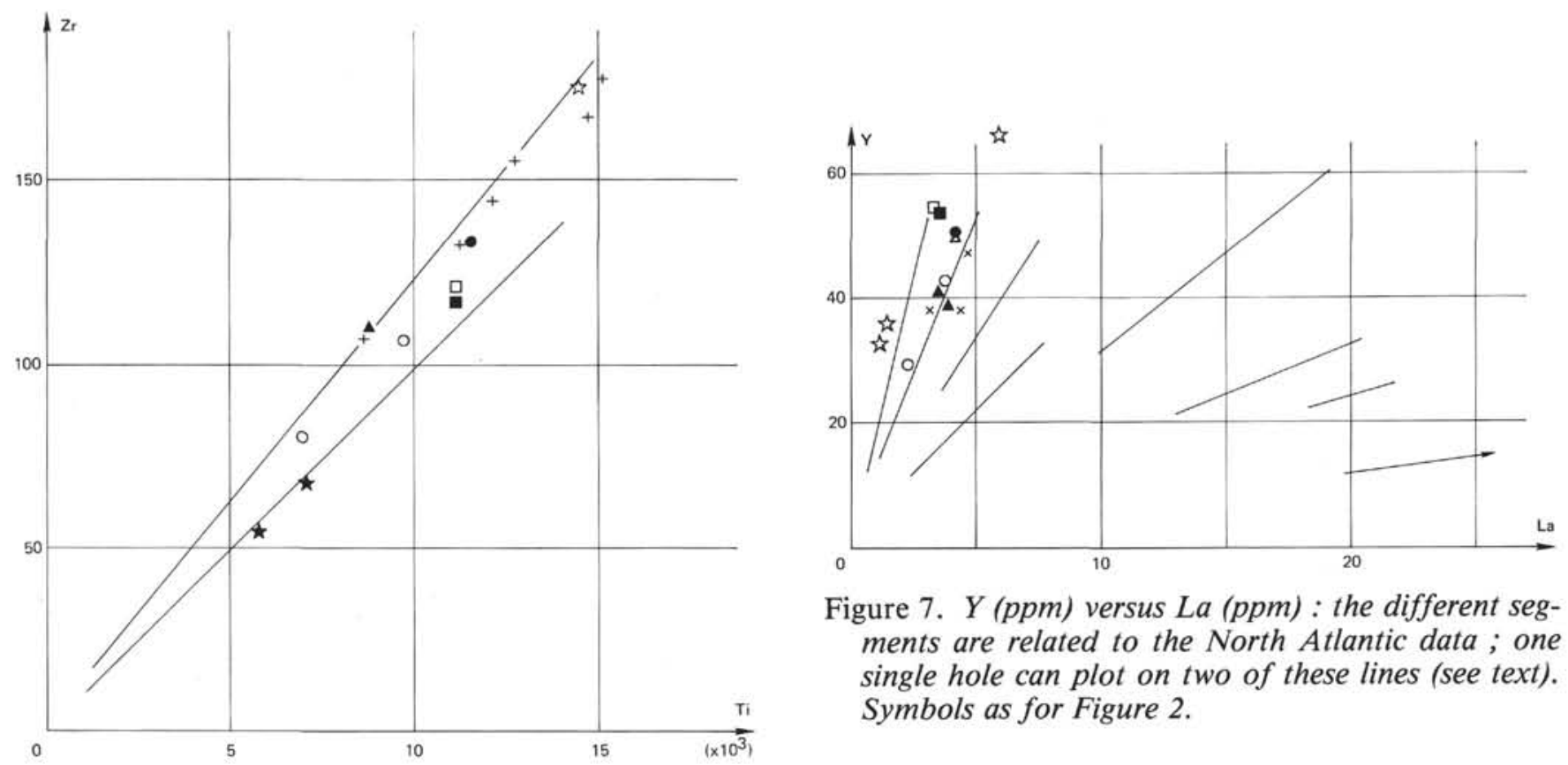

Figure 7. $Y$ (ppm) versus La (ppm) : the different segments are related to the North Atlantic data; one single hole can plot on two of these lines (see text). Symbols as for Figure 2.

Figure 5. $\mathrm{Zr}$ (ppm) versus Ti (ppm) : symbols as for Figure 2. The two lines are a tentative envelope.
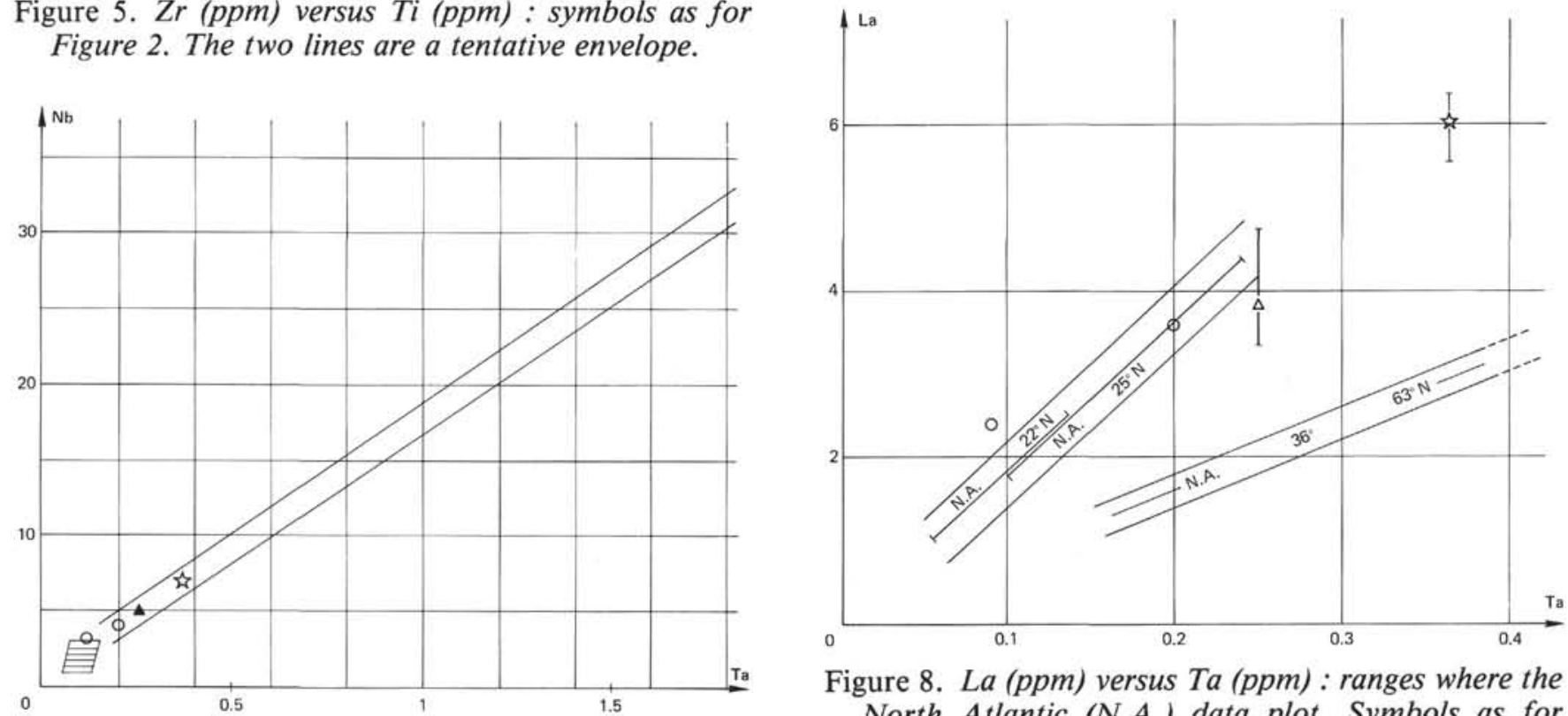

Figure 8. La (ppm) versus Ta (ppm) : ranges where the North Atlantic (N.A.) data plot. Symbols as for Figure 2.

Figure 6. $\mathrm{Nb}(\mathrm{ppm})$ versus $\mathrm{Ta}(\mathrm{ppm})$ : the two parallel lines define the range where the North Atlantic data plot. 目 is specifically related to $25^{\circ} \mathrm{N}$ old crust in the Atlantic (Legs 51 and 52). 